\title{
Hepatic hepcidin/intestinal HIF-2 $\alpha$ axis maintains iron absorption during iron deficiency and overload
}

\author{
Andrew J. Schwartz, ${ }^{1}$ Nupur K. Das, ${ }^{1}$ Sadeesh K. Ramakrishnan, ${ }^{1}$ Chesta Jain, ${ }^{1}$ Mladen T. Jurkovic, ${ }^{1}$ Jun Wu, ${ }^{1,2}$ Elizabeta Nemeth, ${ }^{3}$ \\ Samira Lakhal-Littleton, ${ }^{4}$ Justin A. Colacino, ${ }^{5,6}$ and Yatrik M. Shah ${ }^{1,7}$ \\ 1Department of Molecular and Integrative Physiology, and 'Life Sciences Institute, University of Michigan, Ann Arbor, Michigan, USA. ${ }^{3}$ Department of Medicine, David Geffen School of Medicine, UCLA, \\ Los Angeles, California, USA. ${ }^{4}$ Department of Physiology, Anatomy and Genetics, University of Oxford, Oxford, United Kingdom. ${ }^{5}$ Environmental Health Sciences, ${ }^{6}$ Nutritional Sciences, and ${ }^{7}$ Department of \\ Internal Medicine, Division of Gastroenterology, University of Michigan, Ann Arbor, Michigan, USA
}

\begin{abstract}
Iron-related disorders are among the most prevalent diseases worldwide. Systemic iron homeostasis requires hepcidin, a liver-derived hormone that controls iron mobilization through its molecular target ferroportin (FPN), the only known mammalian iron exporter. This pathway is perturbed in diseases that cause iron overload. Additionally, intestinal HIF-2 $\alpha$ is essential for the local absorptive response to systemic iron deficiency and iron overload. Our data demonstrate a heterotissue crosstalk mechanism, whereby hepatic hepcidin regulated intestinal HIF- $2 \alpha$ in iron deficiency, anemia, and iron overload. We show that FPN controlled cell-autonomous iron efflux to stabilize and activate HIF-2 $\alpha$ by regulating the activity of iron-dependent intestinal prolyl hydroxylase domain enzymes. Pharmacological blockade of HIF-2 $\alpha$ using a clinically relevant and highly specific inhibitor successfully treated iron overload in a mouse model. These findings demonstrate a molecular link between hepatic hepcidin and intestinal HIF-2 $\alpha$ that controls physiological iron uptake and drives iron hyperabsorption during iron overload.
\end{abstract}

\section{Introduction}

More than 1 billion people worldwide are affected by iron overload, iron deficiency, and states of malnutrition that perturb iron homeostasis (1). Diseases of iron overload are among the most common genetic disorders in humans (2). The morbidity and mortality of patients with genetic iron overload are a result of the pathological hyperabsorption of dietary iron, which leads to systemic iron accumulation and iron-induced oxidant damage (3). The master regulator of systemic iron metabolism in mammals is hepcidin, a highly conserved peptide hormone that is predominately synthesized and secreted by the liver. The function of hepcidin is to bind to the only mammalian iron exporter, ferroportin (FPN), resulting in FPN occlusion, internalization from the plasma membrane, and intracellular degradation (4). In states of normal systemic iron and oxygen levels, hepcidin is abundantly produced, FPN is degraded, and iron mobilization into the plasma is limited. Conversely, during iron demand or systemic hypoxia, hepcidin production is repressed to enable FPN stabilization and iron mobilization into the circulation (5). FPN is predominately expressed and regulated in tissues that maintain systemic iron homeostasis, namely, intestine, liver, and macrophages of the reticuloendothelial system (6). Hepcidin-FPN interaction is the essential mechanism by which physiological iron homeostasis is maintained. Genetic mutations that disrupt the hepcidin/FPN axis

\section{Related Commentary: p. 72}

Conflict of interest: The authors have declared that no conflict of interest exists License: Copyright 2019, American Society for Clinical Investigation.

Submitted: May 21, 2018; Accepted: October 16, 2018.

Reference information: / Clin Invest. 2019;129(1):336-348.

https://doi.org/10.1172/JCl122359. give rise to all known forms of iron overload in mammals, referred to as hereditary hemochromatosis $(2,3,5,6)$. These data have fueled much research over the past decade on the molecular mechanisms that regulate hepatic hepcidin production in order to gain insight into how systemic iron homeostasis is maintained.

In addition to hepcidin, local intestinal regulation of iron handling plays an essential role in the maintenance of systemic iron homeostasis. HIF-2 $\alpha$ is also sensitive to cellular iron and oxygen levels as the master intestinal transcriptional regulator of apical and basolateral iron transporters and is essential for maintaining postnatal systemic iron levels $(7,8)$. HIF- $2 \alpha$ is necessary and sufficient to mediate the adaptive increase in iron absorption during both systemic iron deficiency and erythropoietic demand under systemic hypoxia through direct transcriptional activation of the iron absorptive machinery (9-11). HIF-2 $\alpha$ also controls the hyperabsorption of dietary iron that leads to systemic iron accumulation in diseases of iron overload, such as $\beta$-thalassemia and sickle cell disease (12-14). However, the precise molecular cues that initiate and maintain intestinal HIF-2 $\alpha$ during normal physiology and in disease are poorly understood. Moreover, it is unclear whether there is a concerted molecular integration of the systemic hepcidin pathway to local intestinal HIF- $2 \alpha$ signaling in the regulation of iron homeostasis.

This study establishes that intestinal HIF- $2 \alpha$ signaling is regulated by hepatic hepcidin dynamics. Through temporal in vivo and in vitro models of hepcidin and FPN modulation, this work demonstrates that the hepatic hepcidin and intestinal HIF- $2 \alpha$ crosstalk is essential during iron overload, systemic iron deficiency, and anemia. Through unbiased whole-genome RNA-Seq analysis, we demonstrated that the canonical HIF- $2 \alpha$ transcriptional response in the intestine is mediated by hepatic hepcidin. 
Mechanistically, the hepcidin/FPN axis controls HIF- $2 \alpha$ in a cell-autonomous fashion by limiting the activity of iron-dependent prolyl hydroxylase domain (PHD) enzymes. A pharmacological inhibitor of HIF- $2 \alpha$ that is in clinical phase II trials for clear-cell renal cell carcinoma (NCT03108066; ClinicalTrials. gov) demonstrated reversal of iron overload in a mouse model.

\section{Results}

Inducible deletion of hepatic hepcidin leads to the activation of intestinal HIF-2 $\alpha$ and rapid iron accumulation. To understand the molecular connection between hepatic hepcidin and intestinal HIF- $2 \alpha$, mice that express a tamoxifen-inducible $\mathrm{Cre}^{\mathrm{ERT2}}$ fusion protein under the control of the serum albumin promoter $(A l b)$ were crossed with hepcidin 1-floxed (Hamp-floxed) mice (Alb CreERT2 $\left.H a m p^{f l f l}\right)$, giving rise upon tamoxifen administration to mice null for hepatic hepcidin (Hamp $\mathrm{p}^{4 L i v}$ ) (Figure 1A). This inducible model allows for temporal, in vivo study of hepcidin action on HIF- $2 \alpha$, without the confounding effects that arise in later stages of hepcidin deficiency iron overload, namely, the accumulation of ROS (15). Moreover, hepatocyte-specific deletion of hepcidin leaves intact the sources of hepcidin that exist outside of the liver, such as in the heart, where cell-autonomous regulation of cardiac iron homeostasis has recently been shown to exist (16). In this model, the hepcidin transcript Hamp was significantly decreased in livers as early as 2 weeks after tamoxifen treatment (Figure 1B). Interestingly, liver expression of the serum iron uptake receptor transferrin receptor $(T f r c)$ was significantly decreased at 4 weeks, while the FPN transcript (Fpn) was increased at 2 weeks (Supplemental Figure 1A; supplemental material available online with this article; https://doi.org/10.1172/JCI122359DS1). Prussian blue staining revealed progressive liver iron overload. Liver iron accumulation at 2 weeks was minimal, but we detected a time-dependent increase at 4 and 12 weeks. Histological analysis revealed minimal morphological differences across all time points (Figure 1C). An iron assay revealed a significant increase in serum iron as soon as within 2 weeks, with no further increase at 4 or 12 weeks, suggesting that serum iron is rapidly saturated following disruption to hepcidin (Figure 1D). A major complication for patients with hemochromatosis is cardiac dysfunction (17). We found that Prussian blue staining did not detect heart iron accumulation at 2 or 4 weeks following hepcidin deletion but observed significant iron accumulation and disruptions in cellularity and tissue architecture by 12 weeks (Supplemental Figure 1B). We observed similar tissue iron loading in the heart and pancreas (Figure 1, E and F, and Supplemental Figure 1B). In order to assess the regulation of intestinal HIF- $2 \alpha$ by hepatic hepcidin prior to confounding effects that are associated with iron overload, we performed immunohistochemical analysis on duodenal sections 2 weeks after tamoxifen treatment. We detected a robust increase in HIF- $2 \alpha$ protein expression (Figure $1 G$ ). Consistent with these data, we also observed activation of iron-absorptive, HIF- $2 \alpha$-specific target genes and proteins duodenal cytochrome b (Dcytb, also known as Cybrd1), divalent metal transporter 1 (Dmt1, also known as Slc11a2), Fpn, and Ank$r d 37$, as well as the expression of duodenal Tfrc, an indicator of low cellular iron status (Figure 1, H and I) (10). We detected no change in the expression of HIF-1 $\alpha$ target genes (Supplemental Figure 1C) or HIF-2 $\alpha$ inflammatory target genes (Supplemental Figure 1D)
$(18,19)$. Furthermore, we observed no change in HIF-2 $\alpha$-regulated transcripts in the kidneys or spleen, while Tfrc expression was decreased in both organs, suggesting that the hepcidin/HIF- $2 \alpha$ axis was specific to the intestine (Supplemental Figure 2, A and B). These data indicate that changes to hepcidin regulate HIF- $2 \alpha$ stability and activity in physiology and disease.

FPN is necessary for the activation of intestinal HIF-2 $\alpha$ during systemic iron deficiency. To address the molecular mechanism by which hepatic hepcidin regulates intestinal HIF- $2 \alpha$, we investigated the intestinal iron exporter and only target of hepcidin, FPN, in a context of systemic iron demand. Fpn-floxed mice were bred with mice that express a tamoxifen-inducible $\mathrm{Cre}^{\mathrm{ERT} 2}$ fusion protein under the control of the villin ( $\mathrm{Vil}$ ) promoter $\left(\mathrm{Vil} \mathrm{l}^{\mathrm{CrERT2}} \mathrm{Fp} \mathrm{n}^{f l / f)}\right.$ ), giving rise upon tamoxifen administration to mice null for FPN in the intestinal epithelium $\left(F p n^{4 I E}\right) . F p n^{f / / l}$ and $V i l^{C r e E R T 2} F p n^{f / f l}$ mice were placed on a 350ppm (iron-replete) diet or on a diet of less than 5-ppm iron (low-iron) for 1 week, injected with tamoxifen on 3 consecutive days, and sacrificed after an additional week on the respective diets (Figure 2A). This model mimics hepcidin excess at the intestinal level and leads to iron retention in intestinal epithelial cells, despite a state of systemic iron demand (Figure 2B). Duodenal Fpn was significantly decreased following tamoxifen treatment (Figure 2C), and duodenal iron retention was detected by Western blotting for the intracellular iron storage protein ferritin (FTH1) (Figure 2D). We found that hepcidin (Hamp) transcript levels were significantly decreased in mice on the low-iron diet and in $F p n^{\triangle I E}$ mice (Figure 2E). This time point did not induce anemia, as RBC numbers, hemoglobin (HB) counts, hematocrit (HCT) levels, mean corpuscular hemoglobin ( $\mathrm{MCH}$ ) levels, and mean corpuscular volume (MCV) were all unchanged across the cohorts (Figure $2 \mathrm{~F}$ and Supplemental Figure $3 \mathrm{~A}$ ). As complete blood count parameters were unaffected, this model dissociated the enterocyte cellular response to iron deficiency from hypoxia. As expected, we found that duodenal HIF-2 $\alpha$ was stabilized and that HIF- $2 \alpha$-specific ironabsorptive genes were increased in $F p n^{f / f l}$ mice on a low-iron diet. These responses were abrogated in $F p n^{\Delta I E}$ mice (Figure 2, G and $\mathrm{H}$ ). We also observed no changes in the expression of HIF-1 $\alpha$ target genes or HIF-2 $\alpha$ inflammatory target genes (Supplemental Figure 3, B and C). These data demonstrate that the intestinal HIF- $2 \alpha$ response to systemic iron demand occurs downstream of the hepcidin/FPN axis.

FPN is essential for the intestinal HIF-2 $\alpha$ response to erythropoietic demand. Intestinal HIF-2 $\alpha$ is critical for the adaptive increase in iron absorption that enables efficient erythropoiesis $(9,10)$. This increase in erythropoiesus has been postulated to be regulated by changes in intestinal epithelial oxygen levels. We used a phenylhydrazine (Phz) hemolytic anemia model, which stimulates massive erythropoiesis. Fp $n^{f / f l}$ and $V i l^{\text {CreERT2 }} F p n^{f l / f l}$ mice were injected with tamoxifen and allowed to recover for 1 week. Two consecutive injections of either vehicle or Phz were administered, and the mice were sacrificed 48 hours later (Figure 3A). We found that the erythropoietin (Epo) transcript levels were significantly elevated in the kidneys of Phz-treated mice, indicating a state of erythropoietic drive and systemic hypoxia (Figure 3B). Hepcidin (Hamp) transcript levels were significantly decreased in the Phz-treated mice and vehicle-treated $F p n^{4 I E}$ mice (Figure 3C). We found that duodenal ferritin abundance was decreased following Phz treatment in Fpn $n^{f / f l}$ mice, indicating the mobilization of intestinal iron, while this response was blunted in $F p n^{\Delta I E}$ mice (Figure 3D). Interestingly, and consistent with the 
A
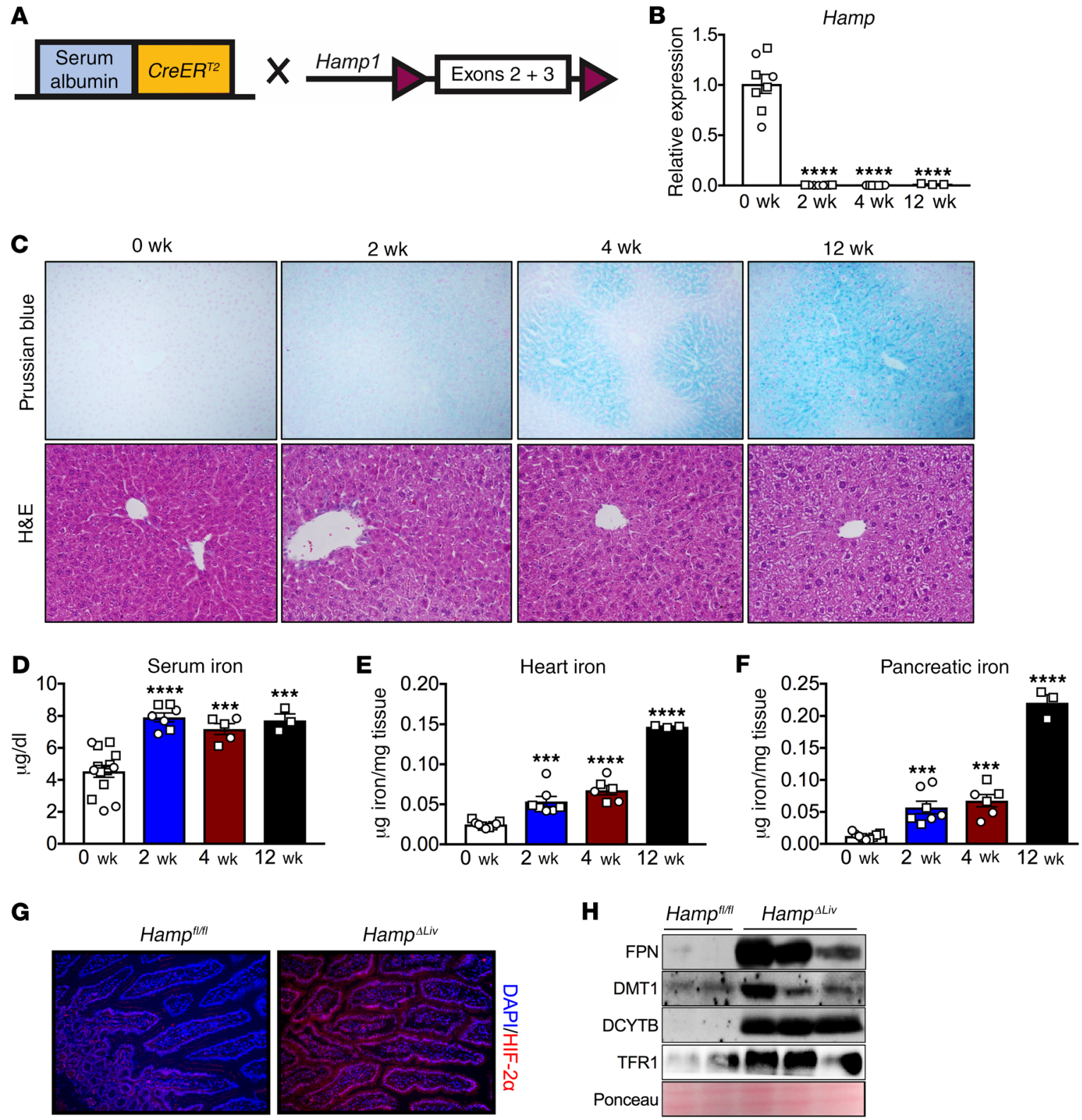

G
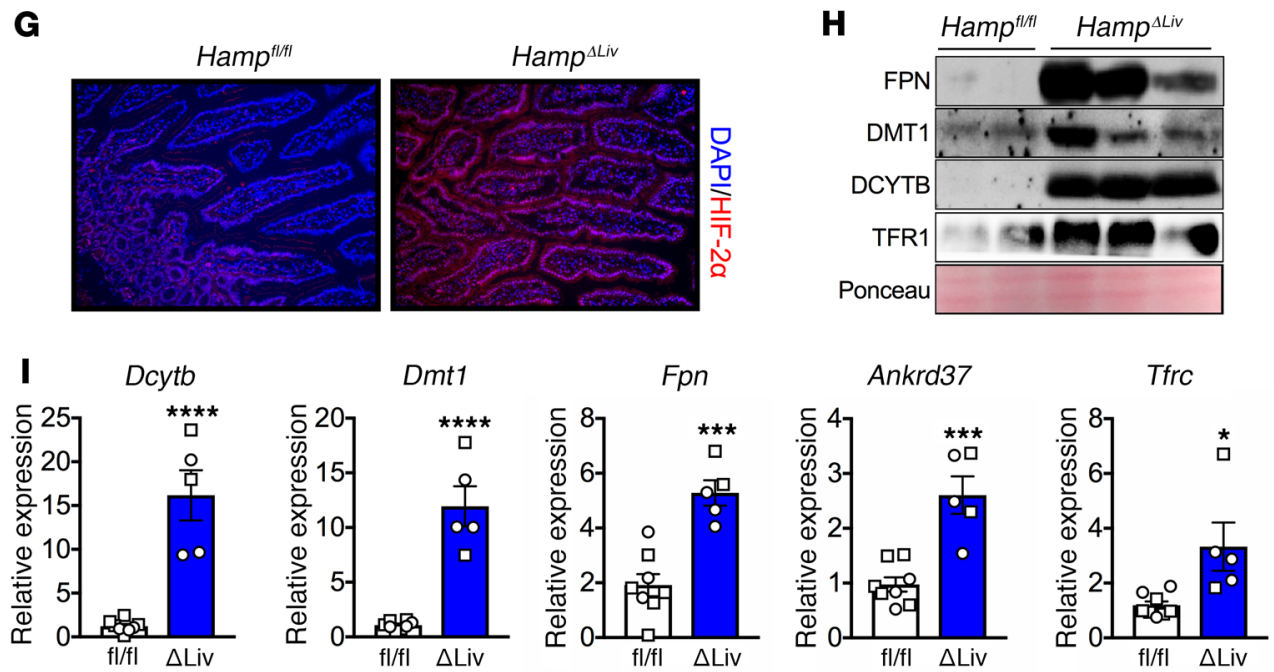

Figure 1. Temporal disruption of hepatic hepcidin activates intestinal HIF-2 $\alpha$ and leads to rapid iron accumulation. (A) Schematic representation of mice with temporal disruption of hepatocyte hepcidin. (B) qPCR analysis of hepatic hepcidin (Hamp) transcript expression levels ( $n=3-8$ per group). (C) Representative Prussian blue iron staining and H\&E staining of liver tissue from Hamp ${ }^{\Lambda L i v}$ mice. Original magnification, $\times 20$ ( $n=3$ per group). (D-F) Serum (D), heart (E), and pancreatic iron content (F) ( $n=3-14$ per group). (C) Representative HIF-2 $\alpha$ staining of duodenal sections 2 weeks after tamoxifen injection into Hamp ${ }^{f l / f l}$ and Hamp ${ }^{\Delta L i v}$ mice. Original magnification, $\times 20$ ( $n=3$ per group). (H) Western blot analysis of FPN, DMT1, DCYTB, and TFR1 expression in duodenal membrane fractions ( $n=2-3$ per group). (I) qPCR antalysis of duodenal HIF-2 $\alpha$-specific and iron-handling transcripts 2 weeks after tamoxifen injection into Hamp $p^{f / f l}$ and Hamp ${ }^{\triangle L i v}$ mice ( $n=5-8$ per group). Data represent the mean \pm SEM. Male samples are designated as squares, and female samples are designated as circles. Significance was determined by 1-way ANOVA with Tukey's post hoc test (B and D-F) or 2-tailed, unpaired $t$ test (I). ${ }^{*} P<0.05,{ }^{* * *} P<0.001$, and ${ }^{* * * *} P<0.0001$ versus the Hamp ${ }^{f / f l}$ group. 
A

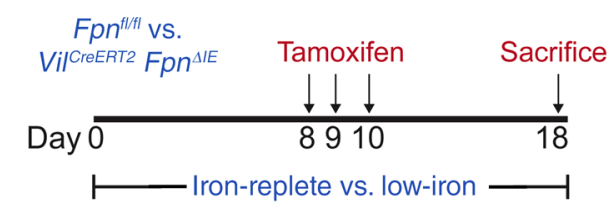

B

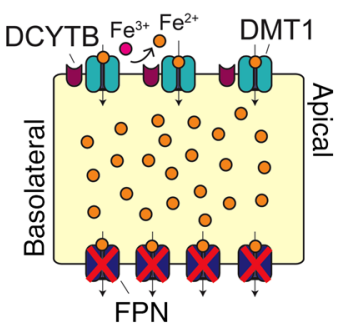

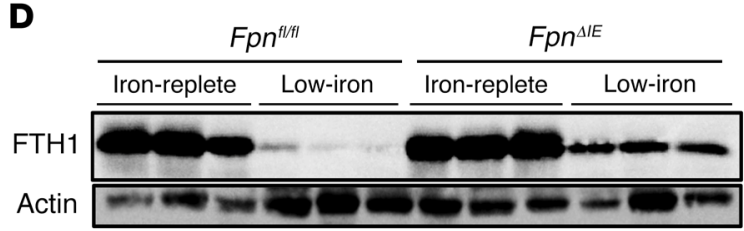

E
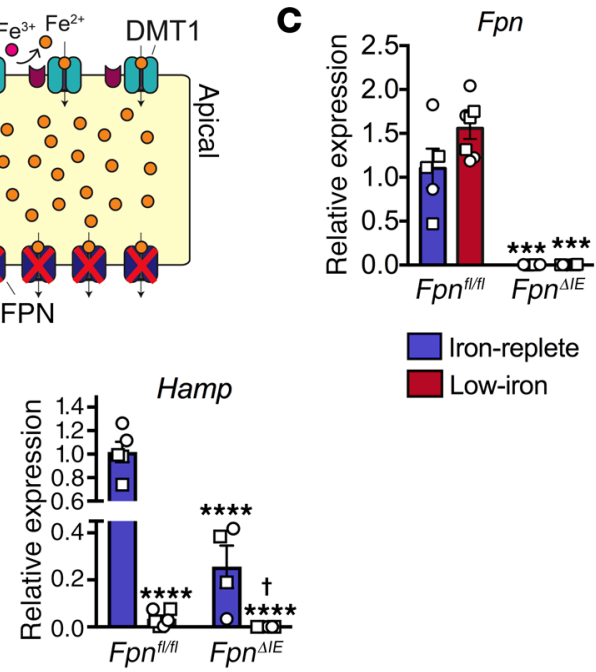
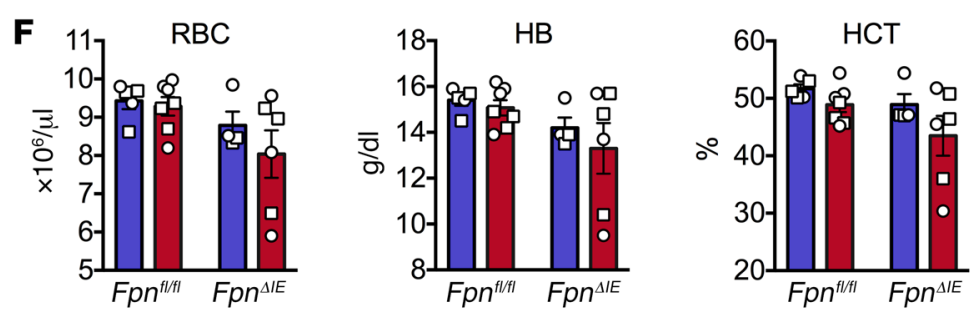

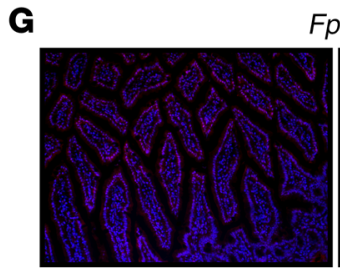

Iron-replete

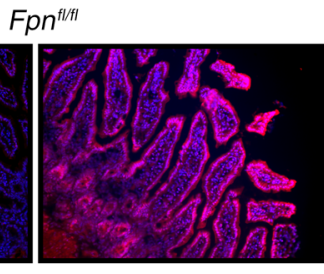

Low-iron

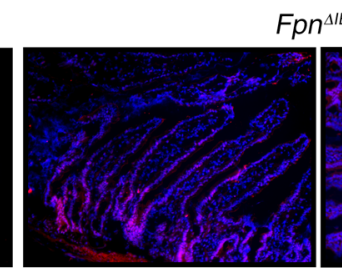

Iron-replete
$F p n^{\Delta I E}$

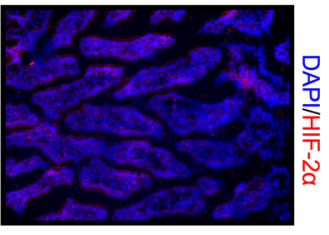

Low-iron
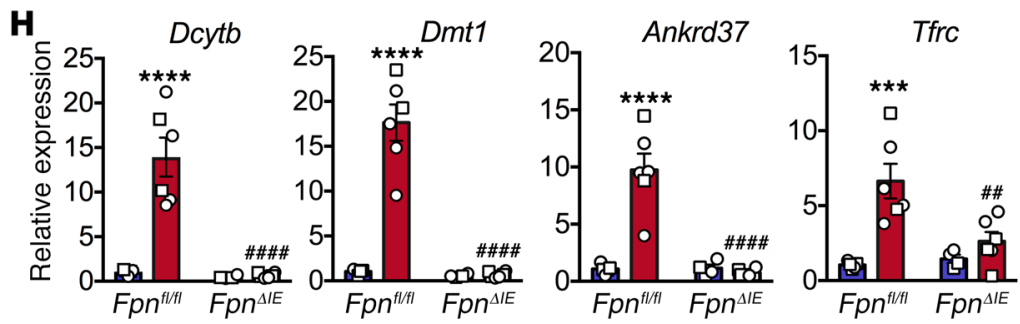

Figure 2. Intestinal epithelial FPN is necessary for the activation of intestinal HIF-2 $\alpha$ during systemic iron deficiency. (A and B) Schematic representation of the experimental design (A) and of intestinal epithelial iron retention following FPN deletion (B). (C) qPCR analysis of duodenal Fpn transcript levels ( $n=4-7$ per group). (D) Western blot analysis of duodenal FTH1 ( $n=3$ per group). (E) qPCR analysis of Hamp transcript levels ( $n=$ 4-7 per group). (F) Analysis of RBC, HB, and HCT ( $n=4-7$ per group). (G) Representative HIF-2 $\alpha$ staining in duodenal sections. Original magnification, $\times 20$ ( $n=3$ per group). (H) qPCR analysis of HIF-2 $\alpha$-specific and iron-handling transcripts in duodenal samples ( $n$ $=4-6$ per group). Male samples are designated as squares, and female samples are designated as circles. Data represent the mean \pm SEM. Statistical significance was determined by 2-way ANOVA with Tukey's post hoc test. ${ }^{* * *} P<0.001$ and ${ }^{* * * *} P<0.0001$ versus iron-replete $F p n^{f l / f l} ;{ }^{\# \# P} P 0.01$ and \#\#\#\# $P<0.0001$ versus low-iron $F p n^{f l / f f}$; ${ }^{t} P<0.05$ versus iron-replete $F p n^{\Delta I E}$. low-iron response, the activation of intestinal HIF- $2 \alpha$ and HIF- $2 \alpha-$ specific iron-absorptive genes during stress erythropoiesis was completely dependent on intact intestinal FPN (Figure 3, E and F). We also detected no change in the expression of HIF-1 $\alpha$ target genes or HIF-2 $\alpha$ inflammatory target genes (Supplemental Figure 4, A and B). These data demonstrate that the hepcidin/FPN axis is essential for the response of intestinal HIF-2 $\alpha$ to low systemic oxygen levels.

Intestinal epithelial iron regulates the HIF-2 $\alpha$ response to changes in systemic iron and oxygen. To clearly demonstrate that enterocyte iron flux was the major mechanism by which the hepcidin/ FPN axis regulated HIF- $2 \alpha$, we sought to modulate luminal versus enterocyte iron levels. To modulate luminal enterocyte levels, Dmt1-floxed mice were bred with mice that express a tamoxifeninducible $\mathrm{Cre}^{\mathrm{ERT} 2}$ fusion protein under the control of the villin (Vil) promoter (Vil CreERT2 $\left.D m t 1^{f l f l}\right)$, giving rise upon tamoxifen administration to mice null for DMT1 in the intestinal epithelium $\left(D m t 1^{I I E}\right)$. Enterocyte iron levels were modulated using Fpn $n^{\triangle I E}$ animals, as explained above. Long-term disruption of DMT1 or FPN gave rise to a state of systemic iron deficiency anemia, with differences seen only in the compartment of iron trapping (i.e., luminal vs. enterocytic iron retention) (Figure 4A). Vil ${ }^{\text {CreERT2 }} \mathrm{Fp}^{f l / f l}, \mathrm{Vil}^{\text {CreERT2 }} \mathrm{DMT} 1^{f l / f l}$, and their littermate controls were assessed 3 months after tamoxifen treatment. We found that Fpn and Dmt1 transcripts levels were significantly decreased in $F p n^{\Delta I E}$ and $D m t 1^{\triangle I E}$ mice, respectively (Figure 4B). Fpn transcript levels were significantly elevated in $D m t 1^{I I E}$ mice, while we detected no change in Dmt1 transcript levels in $F p n^{\Delta I E}$ mice. As expected, the hepcidin (Hamp) transcript was potently repressed in the $F p n^{\triangle I E}$ and $D M T 1^{\Delta I E}$ cohorts as compared 
A

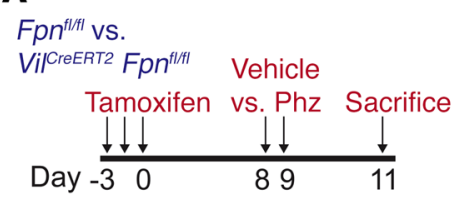

B

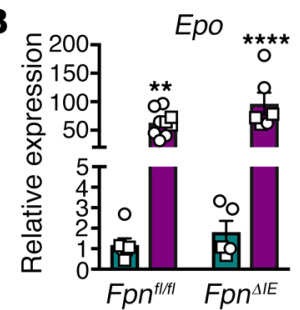

C.

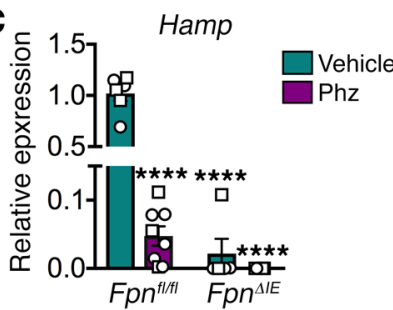

D

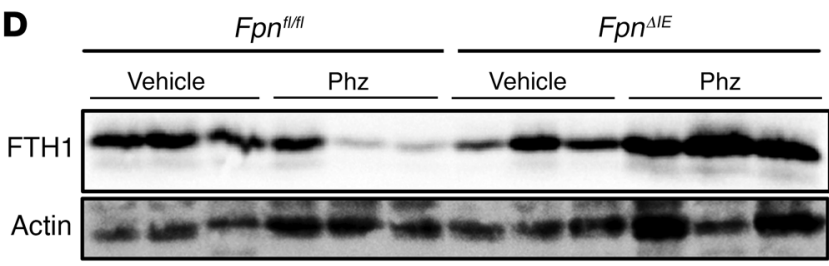

E $F p n^{\text {fith }}$

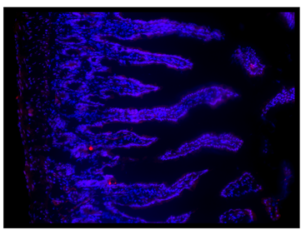

Veh

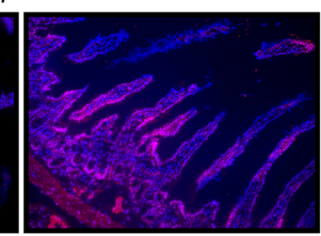

Phz

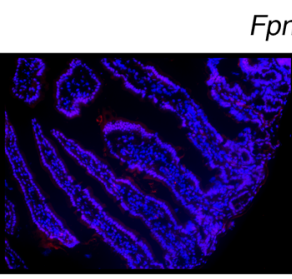

Veh
$F p n^{\Delta I E}$

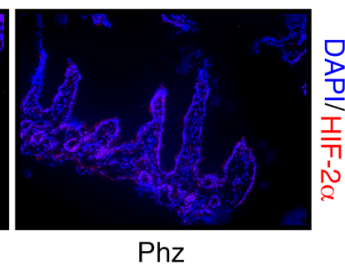

Figure 3. Deletion of intestinal epithelial FPN blocks the intestinal HIF-2 $\alpha$ response to erythropoietic demand.

(A) Experimental design for Phzinduced hemolytic anemia model. (B and C) qPCR analysis of kidney Epo (B) and liver Hamp (C) transcript levels ( $n=5-9$ per group). (D) Western blot analysis of duodenal FTH 1 ( $n=3$ per group). (E) Representative HIF-2 $\alpha$ staining of duodenal sections. Original magnification, $\times 20$ ( $n=3$ per group). (F) qPCR analysis of HIF-2 $\alpha$-specific and iron-handling transcripts in duodenal samples ( $n=5-7$ per group). Male samples are designated as squares, and female samples are designated as circles. Data represent the mean \pm SEM. Statistical significance was determined by 2-way ANOVA with Tukey's post hoc test. ${ }^{*} P<0.05$, ${ }^{*} P<0.01$, and ${ }^{* * *} P<0.0001$ versus vehicle $F p n^{f / / f l} ; \# P<0.05$ and ${ }^{\# \# \# \# P<}$ 0.0001 versus vehicle $F p n^{f / f l}$.
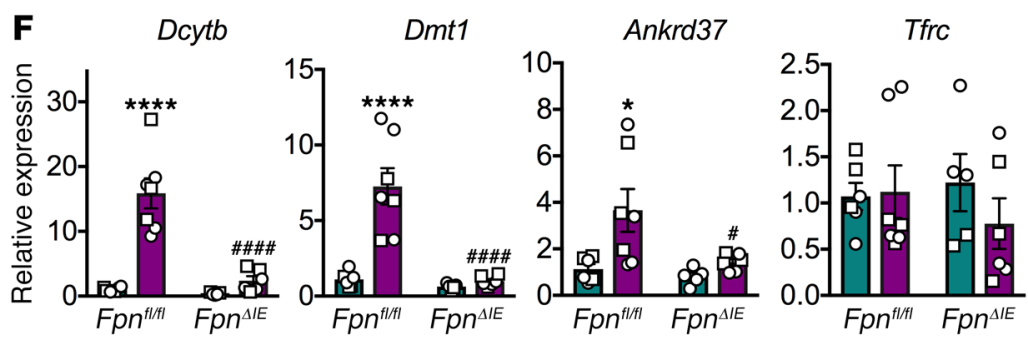

with the $F p n^{f / f l}$ and $D m t 1^{f l / f l}$ mice, respectively (Figure 4C). Furthermore, we detected decreased RBC numbers, HB counts, HCT levels, MCH, and MCV in the Fpn $n^{\triangle I E}$ and $D m t 1^{4 I E}$ mice compared with their littermate controls, which indicated a state of systemic iron deficiency anemia (Figure 4D and Supplemental Figure 5A). Duodenal ferritin abundance was decreased in $D m t 1^{\mathrm{SIE}}$ mice, with the opposite response observed in Fpn $n^{\triangle I E}$ mice (Supplemental Figure 5B). Immunohistochemical analysis revealed significant stabilization of the HIF- $2 \alpha$ protein in $D m t 1^{1 I E}$ mice, with no change in these protein levels in Fpn $n^{4 I E}$ mice (Figure 4E). Moreover, expression levels of the HIF- $2 \alpha$-specific iron genes Dcytb and Ankrd37 were significantly elevated in $D m t 1^{1 I E}$ mice, but not in $F p n^{\Delta I E}$ mice (Figure $4 \mathrm{~F}$ ). We found that Tfrc expression was unchanged in $F p n^{\Delta I E}$ mice and significantly increased in $D m t 1^{4 I E}$ mice compared with expression levels in their littermate controls (Figure 4F). These data convincingly show that intestinal epithelial iron levels regulate HIF-2 $\alpha$ during systemic iron and oxygen deficiency and during iron deficiency anemia.

The intestinal transcriptome during systemic iron demand matches the intestinal response to hepcidin deficiency. The data thus far suggested that the entire intestinal HIF-2 $\alpha$ response to systemic iron and erythropoietic demand was controlled by hepatic hepcidin. However, the outputs for these experiments relied on the mea- surement of canonical HIF- $2 \alpha$ target genes involved in intestinal iron handling. We used an unbiased, high-throughput RNA-Seq approach to compare the duodenal transcriptome during systemic iron demand with that of hepcidin deficiency iron overload (Supplemental Table 2). Hamp $p^{f / f l}$ and $A l b^{\text {CreERT2 }}$ Hamp $^{f / f l}$ mice were placed on iron-replete and low-iron diets and sacrificed 2 weeks after tamoxifen treatment (Figure 5A). Hepcidin transcript levels were significantly decreased in $H a m p^{f / f l}$ mice on a low-iron diet and in both Hamp ${ }^{4 L i v}$ cohorts (Figure 5B). To assess the most significantly changed transcripts when comparing genotype and diet interactions, samples were clustered hierarchically in an unsupervised manner on the basis of the expression of genes that were differentially expressed between conditions at a high-stringency FDR of less than 0.01. We found that the iron-replete Hamp $p^{f / f l}$ samples clustered separately from the iron-replete Hamp ${ }^{4 L i v}$, low-iron Hamp $p^{f / f l}$, and low-iron Hamp ${ }^{A L i v}$ samples (Figure 5C). This demonstrated in an unbiased fashion that the intestinal transcriptomes during systemic iron demand and iron overload were statistically similar to one another, because the 9 treatment samples did not segregate into discrete experimental clusters. We then generated a heatmap, plotting scaled gene expression of the same differentially expressed genes to assess the identity of the genes used for unsupervised hierarchical clustering (Figure 5D). Importantly, we 
A

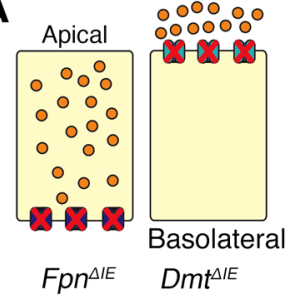

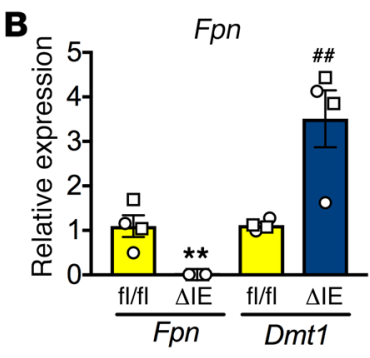
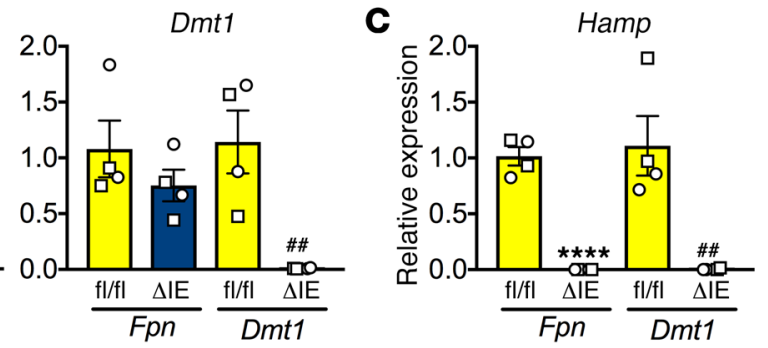

D
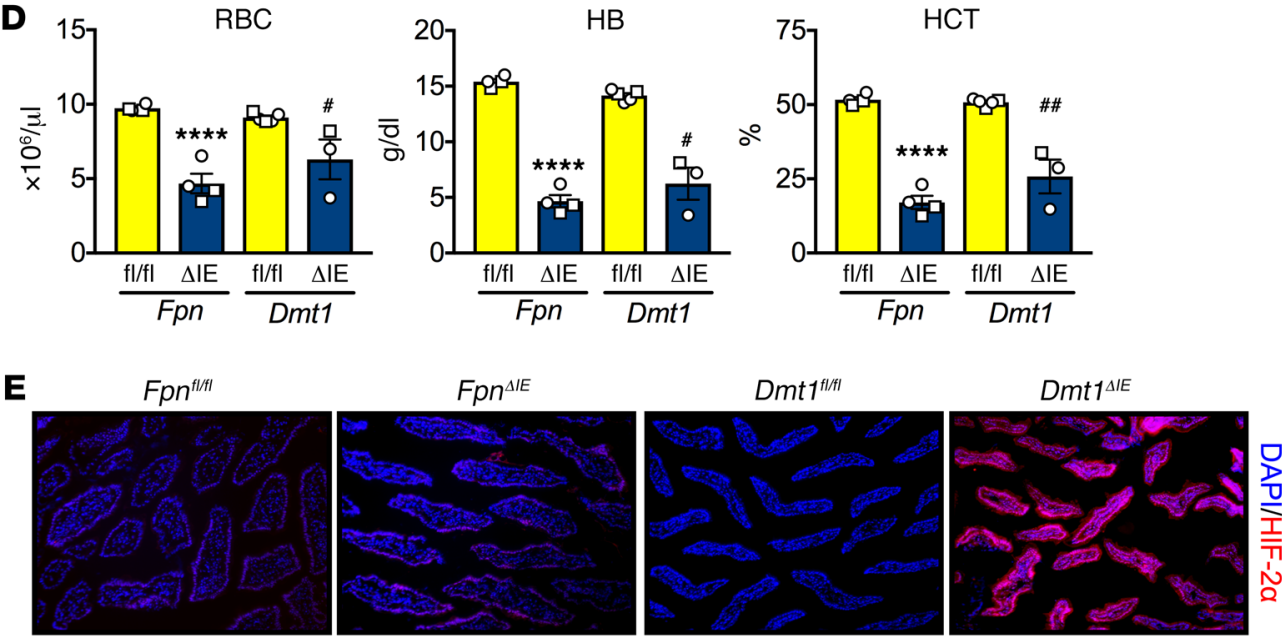

$F$
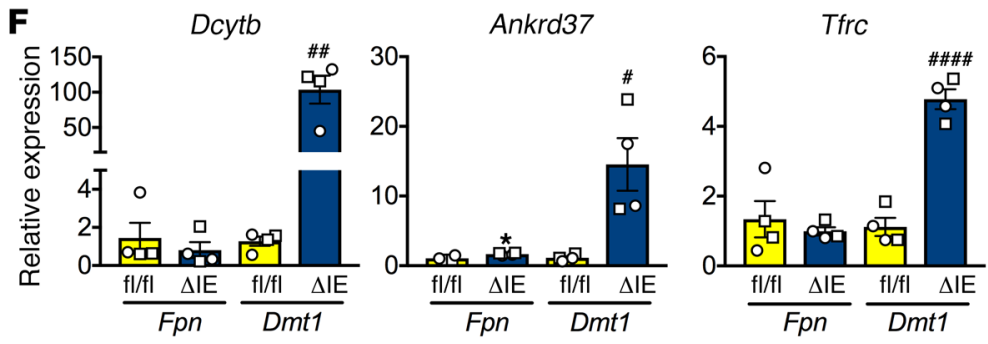

Figure 4. The intestinal HIF-2 $\alpha$ response to changes in systemic iron and oxygen is driven by epithelial iron levels. (A) Schematic of 3-month, inducible iron trapping in animals lacking intestinal epithelial FPN (Fpn $\left.n^{4 \mid E}\right)$ or DMT1 (Dmt $\left.7^{1 / E}\right)$. (B) qPCR analysis of Fpn and Dmt1 transcript levels ( $n=4$ per group). (C) qPCR analysis of hepatic Hamp transcript expression levels ( $n=4$ per group). (D) Analysis of RBC, HB, and HCT ( $n=3-5$ per group). (E) Representative HIF-2 $\alpha$ staining of duodenal sections. Original magnification, $\times 20$ ( $n=3$ per group). (F) qPCR analysis of HIF-2 $\alpha$-specific and iron-handling transcripts in duodenal samples ( $n$ $=4$ per group). Male samples are designated as squares, and female samples are designated as circles. Data represent the mean \pm SEM. Statistical significance was determined by 2 -tailed, unpaired $t$ test. ${ }^{*} P<$ $0.05,{ }^{* *} P<0.01$, and ${ }^{* * * *} P<0.0001$ compared between $\mathrm{Fpn}^{\text {fl/fl}}$ and $\mathrm{Fpn}^{\| / \mathrm{E}}$ cohorts; ${ }^{P} P<0.05,{ }^{\# \# P<0.01 \text {, and }}$ \#\#\#\# $P<0.0001$ compared between $D m t 7^{f / f \mid}$ and $D m t t^{1 / E}$ cohorts. identified the canonical HIF-2 $\alpha$ iron-regulated genes (i.e., Slc11a2, Cybrd1, and Ankrd37). In order to identify novel transcripts in the RNA-Seq data set, we performed a lower-stringency differential expression analysis $(\mathrm{FDR}<0.1)$. Using this approach, we identified genes that were exclusively regulated by iron deficiency (e.g., Nos2, Ccl2O, and Serpine1) and hepcidin deficiency (e.g., Wdr72, $A 4 g n 7$, and $G k n 3$ ), as well as novel target genes regulated in both contexts (e.g., Mir7082, Slc34a2, and Itpr1) (Figure 5E). Collectively, these data demonstrate that the most robustly changed intestinal transcripts during systemic iron demand resemble those in primary hepcidin deficiency iron overload.

FPN activates HIF-2 $\alpha$ in a cell-autonomous manner that is dependent on intracellular iron efflux. To interrogate the molecular mechanism of HIF-2 $\alpha$ stabilization downstream of the hepcidin/FPN axis, we used an in vitro system that models the cellular response to low levels of systemic hepcidin. Most cell lines express very low levels of the FPN protein, and some cell lines appear to be resistant to hepcidin-mediated FPN degradation (10). We assessed hepcidin-sensitive, doxycycline-inducible human FPN ${ }^{\text {GFP }}$ HEK293 cells, as described previously (20). Upon doxycycline treatment, we detected robust FPN ${ }^{\text {GFP }}$ expression by Western blot analysis (Figure
6A). This mimicked a cellular environment of low systemic hepcidin, comparable to that in the intestine, as the fold induction of FPN protein in the FPN ${ }^{\text {GFP }}$ HEK293 cells was similar to that observed in duodenums of Hamp ${ }^{4 L i v}$ mice (Supplemental Figure 6, A and B). To determine whether the regulation of HIF- $2 \alpha$ by hepcidin-FPN is cell autonomous, we treated FPN ${ }^{\text {GFP }}$ cells with doxycycline for 24 hours and generated cytosolic and nuclear fractions. For a positive control, we also treated cells with FG4592, a 2-oxoglutarate analog and chemical inhibitor of the PHD-containing enzymes that regulate HIF. We found that HIF-2 $\alpha$ was robustly stabilized in the nucleus of FPN $^{\text {GFP }}$ cells (Figure 6B). Importantly, HIF-2 $\alpha$ protein was stabilized to the same extent as that seen with FG4592 treatment, suggesting maximal activation. The HIF-2 $\alpha$ response was blunted by iron loading with ferric ammonium citrate (FAC) and recombinant hepcidin treatment, indicating that the activity of HIF-2 $\alpha$ in FPN ${ }^{\text {GFP }}$ cells was dependent on intracellular iron concentration, downstream of hepcidin-mediated FPN degradation (Figure 6C). Together, these data demonstrate cell-autonomous activation of HIF-2 $\alpha$ by iron efflux. This mechanism shows some differences as compared with HIF activation by intracellular iron chelation using compounds such as deferoxamine (DFO), which disrupts mitochondrial function and 
A Hamptitlilvs. Alb CreERT2 $_{\text {Hamp }}^{\text {thlf }}$

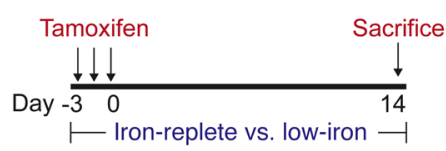

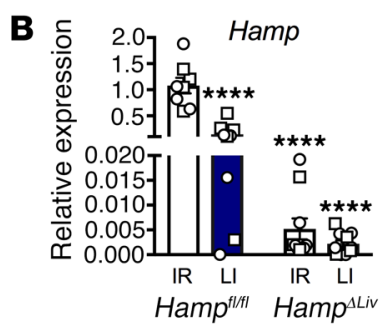
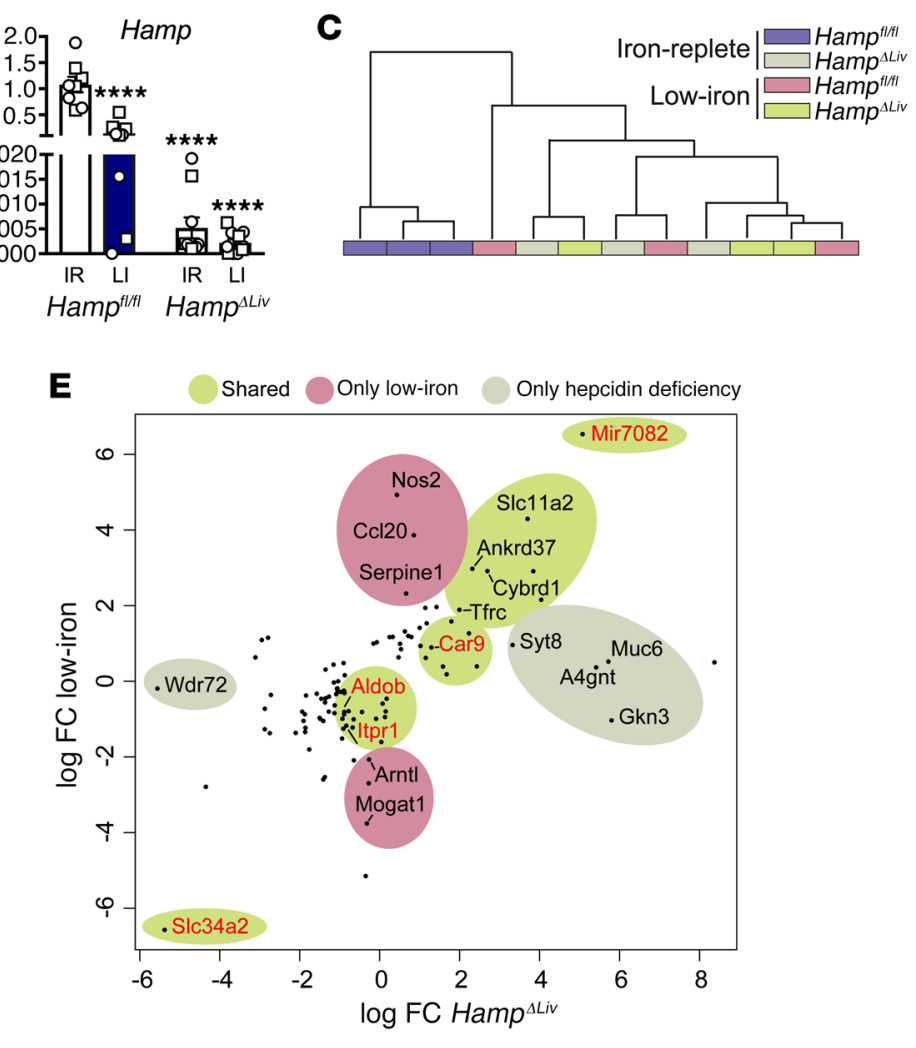

D Iron-replete

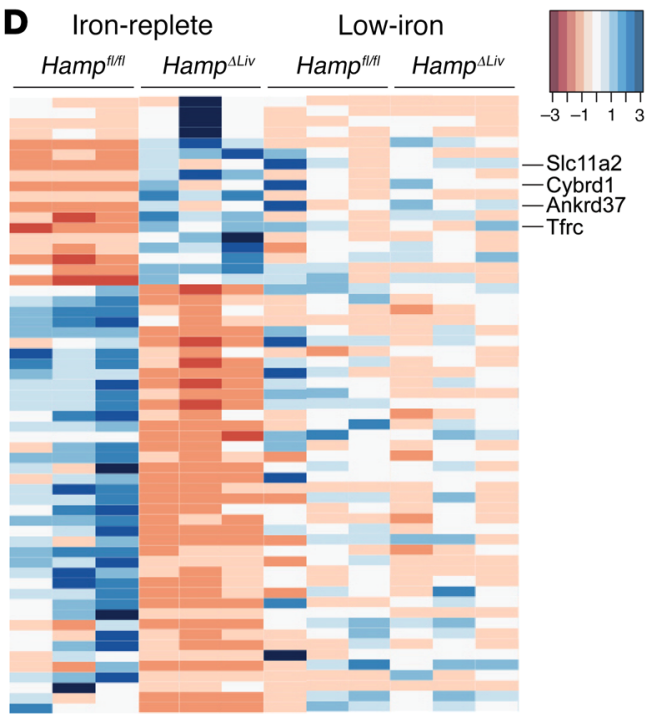

Low-iron

SIC11a2 Ankrd37 Ankrd37

Figure 5. The intestinal transcriptome during systemic iron deficiency resembles that of hepcidin deficiency-mediated iron overload. (A) Experimental design for the samples used in whole-genome RNA-Seq. (B) qPCR analysis of liver Hamp transcript levels in mice on an iron-replete (IR) or low-iron (LI) diet ( $n=8-9$ per group). (C) Dendrogram comparing genotype-diet interactions following unsupervised hierarchical clustering of genes differentially expressed at a high-stringency FDR of less than 0.01 ( $n=3$ per group). (D) Heatmap of genes used for unsupervised hierarchical clustering ( $n=3$ per group). (E) Lower-stringency differential expression analysis at a FDR of less than 0.1 to uncover transcripts in the RNA-Seq data set unique to iron deficiency and hepcidin deficiency. Cenes highlighted in red are novel intestinal transcripts regulated by both low iron and hepcidin deficiency ( $n=3$ per group). Male samples are designated as squares, and female samples are designated as circles. FC, fold change. Data represent the mean \pm SEM. Statistical significance was determined by 2 -way ANOVA with Tukey's post hoc test. ${ }^{* * *} P<0.0001$ versus iron-replete Hamp ${ }^{f / f \prime}$.

results in significant cell death (Supplemental Figure 6C) (21-23). The protein stability of HIF- $2 \alpha$ is regulated by PHD enzymes. We found that PHD enzymes were downstream of hepcidin-FPN in the regulation of HIF- $2 \alpha$, as FG4592 restored the HIF-2 $\alpha$ response in FPN ${ }^{\text {GFP }}$ cells following FAC or recombinant hepcidin treatment (Figure 6D). PHD enzymes require both iron and oxygen for their function. In order to address whether PHD enzyme activity was decreased following FPN stabilization, an adenovirus-based reporter construct to measure PHD enzyme activity was generated by fusing luciferase to a canonical PHD hydroxylation domain (PHD reporter) (Figure 6E). We detected a significant increase in luciferase activity in FPN ${ }^{\text {GFP }}$ cells following doxycycline treatment, and this increase was similar to that seen with chemical inhibition of PHD enzymes by FG4592 treatment (Figure 6F). This response was rescued by loading with FAC and by treatment with recombinant hepcidin. These data demonstrated that stabilization of FPN in the context of low hepatic hepcidin leads to cellular iron efflux, decreased PHD enzyme activity, and, ultimately, cell-autonomous stabilization of HIF- $2 \alpha$. PHD enzymes regulate both HIF- $2 \alpha$ and HIF-1 $\alpha$. However, HIF- $1 \alpha$ protein was stabilized submaximally following FPN overexpression compared with treatment with
FG4592, suggesting selectivity of the hepcidin/FPN axis for HIF-2 $\alpha$ over HIF-1 $\alpha$ (Supplemental Figure 6D). HIF-2 $\alpha$ contains a 5 '-UTR iron-responsive element (IRE) that is responsible for translational inhibition during decreases in intracellular iron (24). Using a HIF$2 \alpha$ IRE luciferase construct, we demonstrated HIF- $2 \alpha$ inhibition following FPN overexpression via doxycycline, with DFO and FAC as controls, suggesting a negative feedback mechanism on FPNmediated activation of HIF-2 $\alpha$ (Supplemental Figure 6E). There are 2 major pools of intracellular iron: (a) labile "free" iron, and (b) iron bound by the intracellular iron storage protein ferritin. The mobilization of ferritin-bound iron requires the lysosomal degradation of ferritin via the rate-limiting cargo protein nuclear receptor coactivator 4 (NCOA4) $(25,26)$. To address which pool of iron is limited for PHD enzymes by FPN, 2 unique NCOA4-KO cell lines were generated and sequence verified in FPN ${ }^{\mathrm{GFP}}$ cells. FPN overexpression led to ferritin degradation in an NCOA4-dependent manner (Figure 6G). However, NCOA4 deletion did not prevent the decreased PHD enzyme activity following FPN overexpression (Supplemental Figure 6F). While doxycycline-inducible FPN ${ }^{\text {GFP }}$ HEK293 cells have been widely used to study hepcidin-FPN dynamics (20, 27, 28), we sought to interrogate the hepcidin/FPN/HIF-2 $\alpha$ axis in an intesti- 
A $\begin{aligned} \text { Doxycycline } & - \\ \text { GFP } & + \\ \text { GAPDH } & \end{aligned}$
B

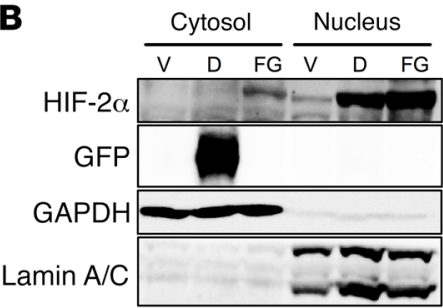

C

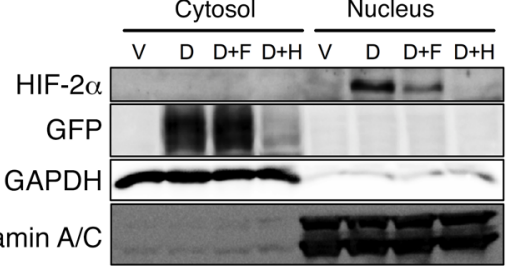

E

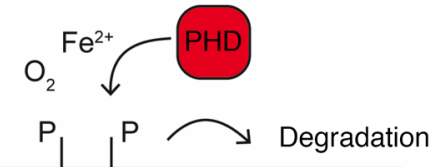

Hydroxylation domain

\section{Luciferase}

Lamin A/C

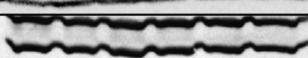

G
$\mathbf{F}$

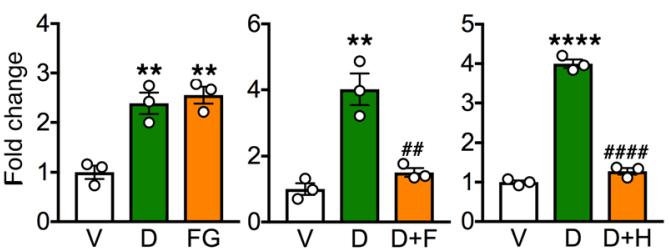
Doxycycline \begin{tabular}{c} 
Empty \\
\hline$-\quad-\quad-\quad+\quad+\quad+$
\end{tabular} NCOA4 sg 1

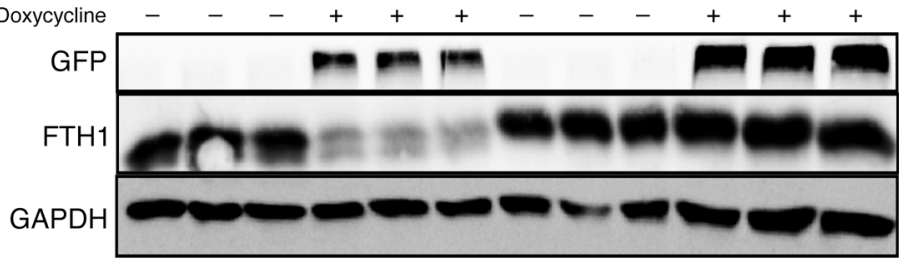$$
\text { GAPDH }
$$

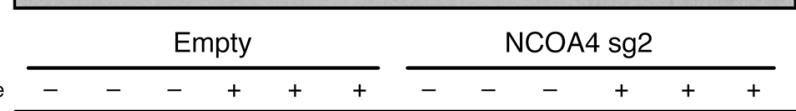

H Doxycycline

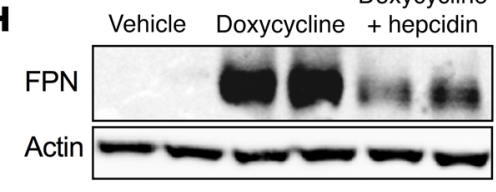

Doxycycline

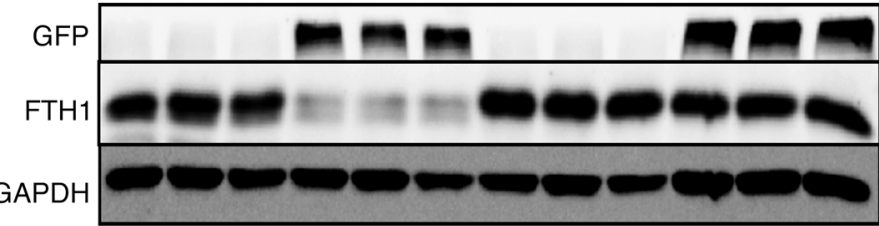

I

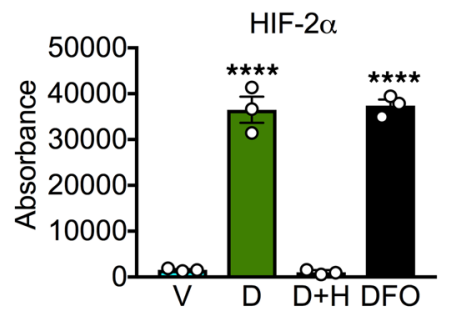

J

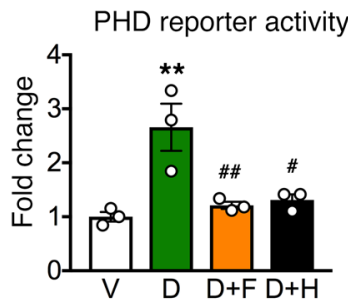

Figure 6. FPN activates HIF-2 $\alpha$ in a cell-autonomous manner that is dependent on efflux of the cellular labile iron pool. (A) Western blot analysis of FPN ${ }^{\mathrm{CFP}}$ HEK293 cells following a 24-hour doxycycline treatment. (B) Western blot analysis of cytosolic and nuclear fractions of FPN ${ }^{\mathrm{CFP}}$ HEK293 cells treated with vehicle (V), $250 \mathrm{ng} / \mathrm{ml}$ doxycycline (D), or $100 \mu \mathrm{M}$ FG4592 (FC) for 24 hours. (C and D) Western blot analysis of cytosolic and nuclear fractions of FPN ${ }^{\mathrm{CFP}}$

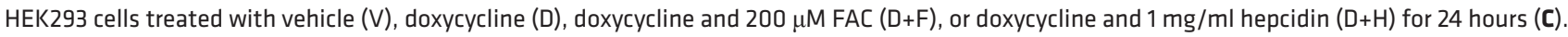
Separate doxycycline plus FAC and doxycycline plus hepcidin conditions were also cotreated with FC4592 for 24 hours, as indicated (D). (E) Schematic of the luciferase-based PHD enzyme activity reporter. (F) Fold change of luciferase activity in FPN ${ }^{\text {FFP }}$ HEK293 cells infected with the PHD reporter and treated with vehicle, doxycycline, FG4592, FAC and doxycycline, or doxycycline and hepcidin for 24 hours. (C) Western blot analysis of FPNCFP HEK293 cells stable for empty lentiCRISPRv2 (Empty) or unique NCOA4 short guide RNAs (NCOA4 sg1 and NCOA4 sg2). Cells were treated with FAC for 24 hours and then with

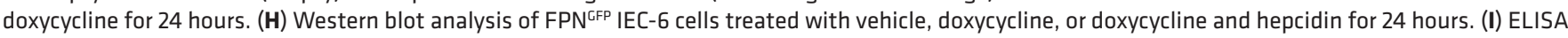

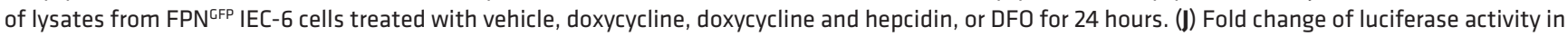
FPN ${ }^{\text {CFP IEC }} 6$ cells infected with the PHD reporter and treated with vehicle, doxycycline, FAC and doxycycline, or doxycycline and hepcidin for 24 hours. All cell culture experiments were repeated at least 3 times. Data represent the mean \pm SEM. Statistical significance was determined by 1-way ANOVA with Tukey's post hoc test. ${ }^{* *} P<0.01$ and ${ }^{* * * *} P<0.0001$ versus vehicle; ${ }^{\#} P 0.05,{ }^{\# \#} P<0.01$, and ${ }^{\# \# \#} P<0.0001$ versus doxycycline. 
nal epithelial cell line. IEC-6 cells are a normal rat small intestinal cell line, and a doxycycline-inducible human FPNGFP IEC-6 cell line was generated. FPN ${ }^{\text {GFP }}$ IEC- 6 cells showed FPN stabilization after doxycycline treatment to a degree similar to that observed in duodenums of Hamp ${ }^{\Lambda L i v}$ mice (Supplemental Figure 6B). Moreover, we found that FPNGFP IEC- 6 cells were highly sensitive to hepcidin (Figure 6H). Several HIF-2 $\alpha$ antibodies that were tested did not detect a specific HIF-2 $\alpha$ band by Western blot analysis of rat lysates (data not shown). We used an ELISA approach with a HIF-2 $\alpha$ antibody that could detect native recombinant HIF- $2 \alpha$. We observed robust HIF- $2 \alpha$ stabilization following FPN overexpression via doxycycline treatment and found that cotreatment with doxycycline and hepcidin completely rescued this response, with DFO serving as a positive control (Figure 6I). Similar to what was observed in vivo, we detected no increase in HIF- $1 \alpha$ following FPN overexpression in the IEC- 6 FPN $^{\text {GFP }}$ cells, while DFO treatment significantly increased HIF-1 $\alpha$ expression, further indicating a difference in the mechanism of action of iron efflux through FPN and iron chelation by DFO (Supplemental Figure 6G). The mechanism of HIF-2 activation by FPN overexpression in IEC- 6 cells was the same as in HEK293 cells, as PHD enzyme activity was decreased by FPN overexpression in an iron- and hepcidin-dependent manner (Figure 6J). Collectively, these data demonstrate that in the absence of hepcidin, stabilization of membrane FPN regulates HIF- $2 \alpha$ in a cell-autonomous manner by depleting the cellular labile iron pool and limiting the activity of PHD enzymes.

Inhibition of HIF-2 $\alpha$ with PT2385 decreases systemic iron accumulation in hepcidin-deficient iron overload. Current therapeutic approaches for patients with iron overload rely on iron chelators and phlebotomy, which lead to significant off-target effects and cause fatigue. We sought to determine whether the hepatic hepcidin/intestinal HIF-2 axis can be therapeutically targeted to treat iron overload. The HIF- $2 \alpha$-specific inhibitor PT2385 was recently developed (29). PT2385 binds to HIF-2 $\alpha$ and prevents its heterodimerization with aryl hydrocarbon receptor nuclear translocator (ARNT), thus preventing the transcriptional activity of HIF-2 $\alpha$ (29). PT2385 is currently in a phase II clinical trial for the treatment of clear-cell renal cell carcinoma (NCT03108066; ClinicalTrials. gov). Hamp $p^{f / f l}$ and $A l b^{\text {CreERT2 }} \mathrm{Hamp}^{f / f l}$ mice were injected with tamoxifen, and 2 weeks later, the Hamp ${ }^{\lrcorner L i v}$ mice were orally gavaged with vehicle or PT2385 daily for 2 weeks (Figure 7A). We observed no change in BW during the treatment period (Supplemental Figure 7A). The hepcidin transcript levels were decreased in both Hamp ${ }^{\Delta L i v}$ cohorts (Figure 7B). Decreased intestinal iron absorption in mice with a genetic disruption of intestinal HIF-2 $\alpha$ leads to anemia (10). Thus, we sought to determine whether prolonged treatment with PT2385 would lead to systemic anemia. We found that kidney Epo transcript levels were decreased in vehicle-treated Hamp $p^{4 L i v}$ mice, while this decrease was abrogated in PT2385-treated Hamp ${ }^{4 L i v}$ mice (Figure 7B). We noted a significant expansion of RBC numbers and increased $\mathrm{HB}$ and HCT in vehicle-treated Hamp ${ }^{\Lambda L i v}$ mice, and these increases were rescued in the PT2385 cohort (Figure 7C). MCV and $\mathrm{MCH}$ levels were unchanged among all groups (Supplemental Figure 7B). Membrane stabilization of the HIF-2 $\alpha$ iron-absorptive targets FPN, DMT1, and DCYTB was elevated in the Hamp $p^{4 L i v}$ mice but was completely absent in the PT2385-treated Hamp ${ }^{\Lambda L i v}$ mice (Figure 7D). Prussian blue staining for iron in the liver was decreased in the PT2385-treated Hamp ${ }^{4 L i v}$ mice compared with that seen in the vehicle-treated mice (Figure 7E). Additionally, quantitative iron assays revealed significant decreases in serum, liver, and pancreatic iron content, with a trend toward a decrease of iron in the heart in PT2385-treated Hamp $p^{4 L i v}$ mice (Figure 7F). These data show that HIF- $2 \alpha$ is a potential pharmacological target downstream of the hepcidin/FPN axis in patients with iron overload (Figure 7G).

\section{Discussion}

Systemic iron homeostasis requires multiple organs working in concert to maintain cellular iron concentrations for metabolism and RBC levels for systemic oxygen transport. Research over the past decade has shown that this system is centrally regulated by the liver-derived hormone hepcidin and requires intestinal iron absorption for the maintenance of postnatal systemic iron levels. However, a complete biological link between the liver and intestine during iron deficiency and in diseases of iron overload has remained unclear. The present work demonstrates that the liver controls the intestine through a hepatic hepcidin/intestinal HIF$2 \alpha$ axis that regulates physiological iron uptake during systemic iron deficiency and drives pathological iron absorption during iron overload caused by hepcidin deficiency. Paradoxically, using unbiased, high-throughput RNA-Seq, we show that the intestinal response to systemic iron deficiency and hepcidin deficiencymediated iron overload is largely the same. The physiological repression of hepcidin during iron demand and the perturbation of hepcidin during genetic iron overload directly trigger iron efflux through intestinal FPN to limit the activity of iron-dependent PHD enzymes. This stabilizes intestinal HIF- $2 \alpha$ to activate genes that are necessary and sufficient for intestinal iron absorption. Interestingly, HIF-2 $\alpha$ activation downstream of hepcidin was intestine specific, as HIF-2 $\alpha$-dependent transcript levels were unchanged in the kidney and the spleen following hepatic hepcidin deletion. Potential explanations include the presence of intestine-specific coactivators and/or genetic suppressors and enhancers. Consistent with data showing that HIF- $2 \alpha$ is necessary for the adaptive increase in intestinal FPN during iron deficiency (10), the present data suggest a feed-forward loop, whereby intestinal FPN stabilization following a decrease in hepcidin activates HIF- $2 \alpha$ to maintain FPN transcript levels during systemic iron demand and in iron overload. Although the hepcidin/FPN/HIF-2 $\alpha$ axis is the major trigger for the intestinal transcriptional response following iron demand, the discovery of smaller subsets of genes that are either regulated by systemic iron deficiency or hepcidin deficiency indicates differences in the intestinal response to a decrease in dietary iron compared with iron hyperabsorption during iron overload. A recent report indicated that the HIF response can be modulated by microbiota-derived short-chain fatty acids, which could partly explain the difference between luminal and systemic cues to the intestine (30). More work needs to be done to understand the regulation of this small subset of genes.

Previous studies showed a critical role for HIF- $2 \alpha$ in the hyperabsorption of iron in primary and secondary hemochromatosis (12-14). However, the field has relied largely on germline knockout strategies to study hepcidin disruption, which gives rise to iron loading that begins during embryonic development and can cause ROS that are known inducers of HIF. Furthermore, full-body hep- 
A

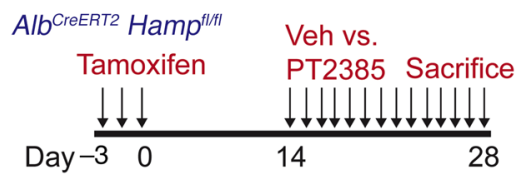

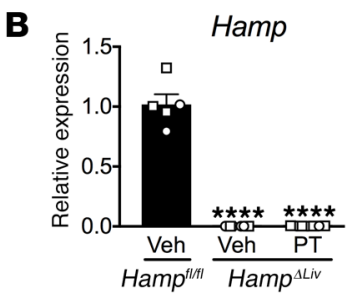
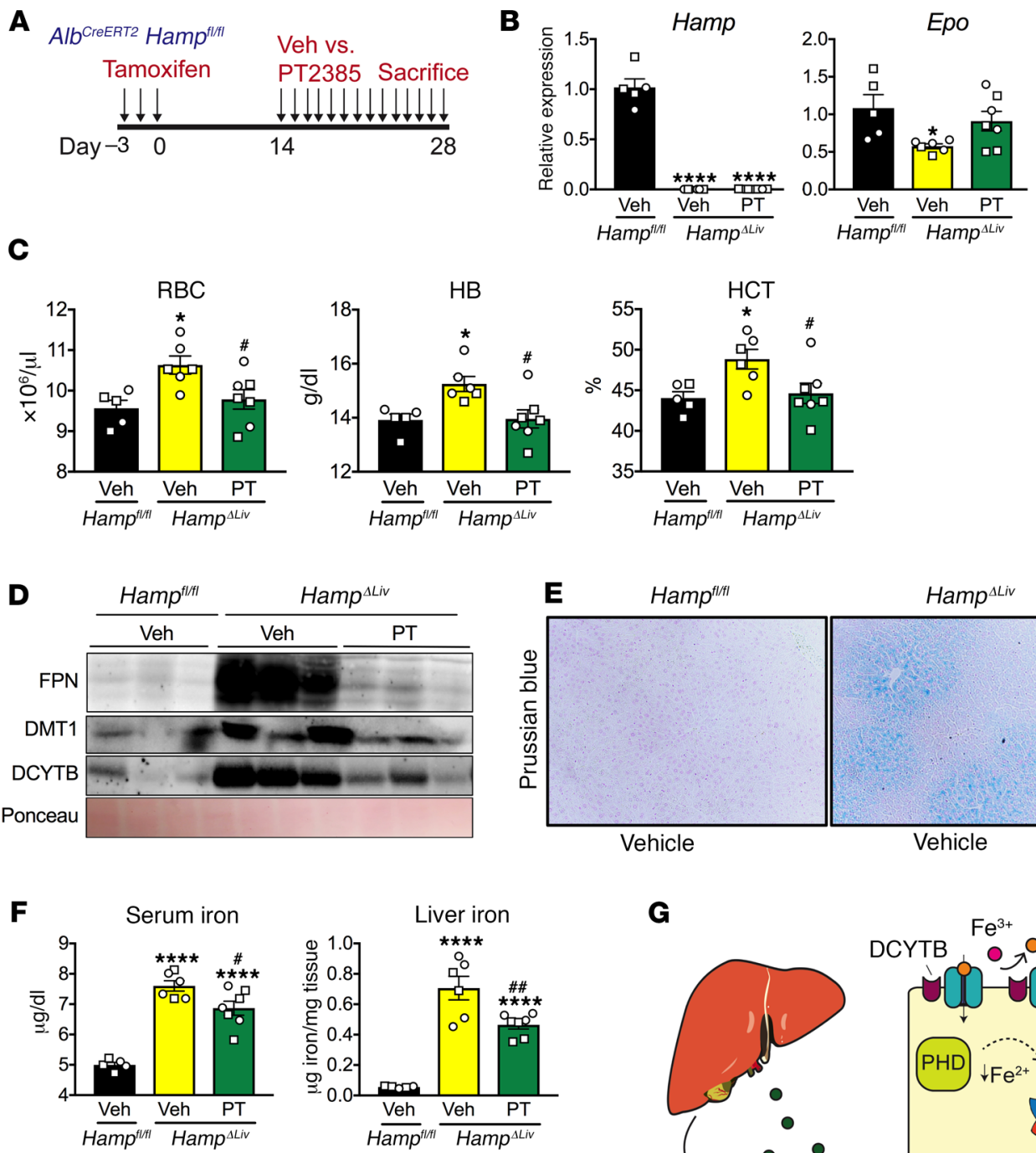

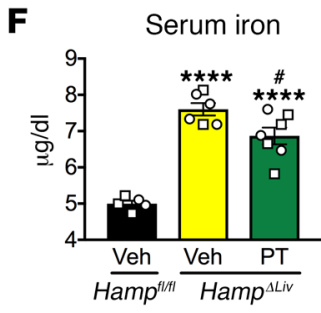

Heart iron

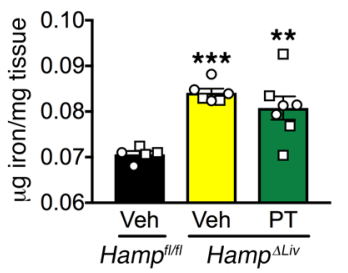

E

Hampitit

Hamp $^{\Delta L i v}$

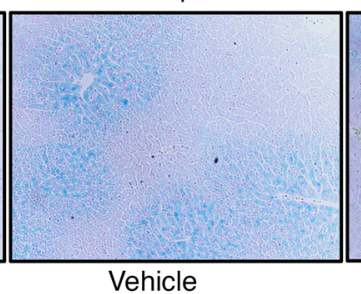

Vehicle
G

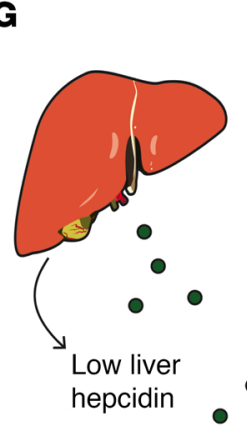

$H_{a m p}{ }^{\Delta L i v}$

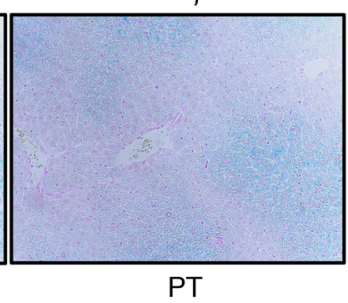

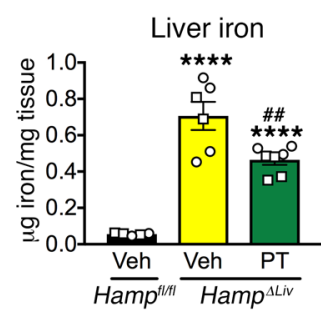

Pancreatic iron

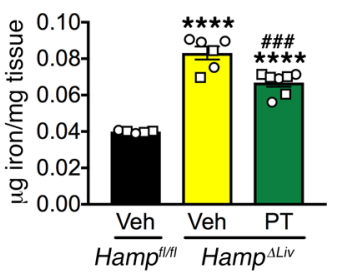

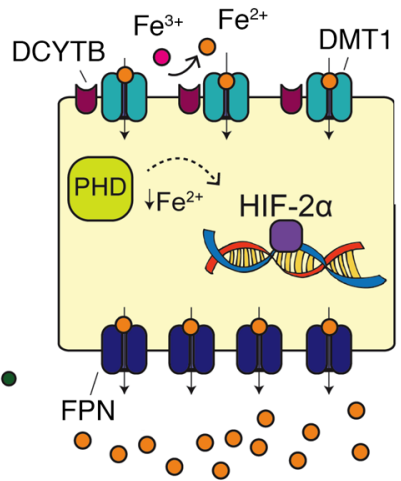

Figure 7. Inhibition of HIF-2 $\alpha$ using PT2385 reverses iron accumulation in multiple tissues in hepcidin-deficient hemochromatosis. (A) Experimental design for oral gavage of vehicle or PT2385 in Hamp ${ }^{4 L i v}$ mice. (B) qPCR analysis of hepatic Hamp and kidney Epo transcript levels ( $n=5-7$ per group). (C) Analysis of RBC, HB, and HCT ( $n=5-7$ per group). (D) Western blot analysis of FPN, DMT1, and DCYTB in duodenal membrane fractions ( $n=3$ per group). (E) Representative Prussian blue staining for iron in liver tissues. Original magnification, $\times 20$ ( $n=3$ per group). (F) Serum, liver, heart, and pancreatic iron content ( $n=5-7$ per group). (G) Schematic representation of hepatic hepcidin/intestinal HIF-2 axis. Male samples are designated as squares, and female samples are designated as circles. Data represent the mean \pm SEM. Statistical significance was determined by 1-way ANOVA with Tukey's post hoc test. ${ }^{*} P<0.05$, ${ }^{*} P<0.01$, ${ }^{* * *} P<0.001$, and ${ }^{* * * *} P<0.0001$ versus vehicle Hamp ${ }^{f / f f}$; $P<0.05$, \#\# $P<0.01$, and ${ }^{\# \# \#} P<0.001$ versus vehicle Hamp ${ }^{4 L i v}$. PT, PT2385; Veh, vehicle.

cidin deficiency disrupts sources of hepcidin outside of the liver that have recently been shown to establish a cell-autonomous mechanism of local iron regulation, particularly in the heart, an organ critical for systemic oxygen transport (31). The use of our inducible model of hepatic hepcidin deletion has characterized, for the first time to our knowledge, the kinetics by which iron overload progresses. There are data to show that iron overload is toxic to RBC survival at later stages of hemoglobinopathies $(12,32)$, while our data demonstrate that there is a significant expansion of the RBC pool in early stages of iron overload, which occurs in a HIF- $2 \alpha$-dependent manner as PT2385 treatment rescues this response. Recent work has revealed erythroid-derived factors that regulate hepcidin to facilitate erythropoiesis (33-35). The present data conversely suggest that hepcidin restricts the 
RBC pool, potentially by limiting intestinal iron absorption or through the direct regulation of signaling downstream of FPNmediated cellular iron efflux in other organs and cell types. Future studies will need to determine the mechanism by which this RBC expansion occurs and whether it is a physiologically relevant process of iron storage during early iron overload.

The dioxygenase superfamily of PHD enzymes regulate the protein stability of both HIF- $2 \alpha$ and HIF- $1 \alpha$. However, our laboratory, among others, has demonstrated that intestinal HIF- $2 \alpha$, but not HIF- $1 \alpha$, is stabilized, transcriptionally active, and necessary and sufficient for iron absorption during systemic iron demand ( 7 , 8). Recent reports have demonstrated that certain PHD isoforms, namely PHD3, show selectivity for HIF-2 $\alpha$ over HIF-1 $\alpha$ (36). Furthermore, small intestinal HIF- $2 \alpha$ is more sensitive to pharmacological inhibition of all PHD isoforms than is HIF-1 $\alpha$ (37). This selectivity could explain the differential activation of small intestinal HIF- $2 \alpha$ over HIF- $1 \alpha$ downstream of hepcidin/FPN/PHDs. Future work will need to establish the Km value of intestinal PHD enzymes for iron to determine whether iron efflux through FPN limits the activity of a HIF- $2 \alpha$-specific PHD.

In addition to hepcidin and HIF- $2 \alpha$, another mammalian iron-sensing axis exists via iron-regulatory protein (IRP) and IRE machinery. This system modulates translation via the binding of IRPs with IREs that exist in the 5'- or 3'-UTR of target transcripts involved in cellular iron handling. Duodenal enterocytes produce a FPN transcript that evades IRP-mediated repression in settings of low intracellular iron by lacking an IRE (38). This variant might function alongside HIF- $2 \alpha$-mediated transcriptional upregulation of Fpn to maintain FPN protein levels following intestinal iron efflux. Interestingly, IRP1 is activated following decreases in intracellular iron to negatively regulate HIF- $2 \alpha$ translation via action on an IRE in the $5^{\prime}$-UTR of the HIF- $2 \alpha$ mRNA, which was shown both in vitro (24) and in vivo (39-41). Recently, this pathway was shown to be pharmacologically targeted to treat HIF-2 $\alpha$-induced polycythemia (42). We also observed repression of the HIF- $2 \alpha$ IRE in our in vitro model following $\mathrm{FPN}^{\mathrm{GFP}}$ stabilization. Taken together, these data show that the hepcidin/FPN/PHD axis may control HIF- $2 \alpha$ during systemic iron deficiency and that IRP1-mediated repression of HIF- $2 \alpha$ translation may limit the level of activation. More work will need to be done to fully understand the interaction between the IRP and IRE systems and the hepcidin/FPN/PHD/ HIF- $2 \alpha$ axis during systemic iron demand.

Iron chelators have been shown for decades to regulate HIF, although these molecules dramatically disrupt mitochondrial function and can strip iron from iron-containing proteins. To our knowledge, these data are the first to show that cell-autonomous, biologically relevant iron efflux regulates intestinal HIF- $2 \alpha-$ mediated iron absorption in vivo, in contexts of both systemic iron deficiency and low systemic oxygen. The present work shows that the FPN-mediated efflux of iron is a cell-autonomous trigger to stabilize HIF- $2 \alpha$. Moreover, this finding demonstrates in vivo that a liverderived endocrine signal plays an essential role in the activity of intestinal enzymes that regulate HIF- $2 \alpha$. Numerous reports have recently begun to characterize the function of FPN in organs that do not play a role in maintaining systemic iron homeostasis $(16,43,44)$. It will therefore be vital to determine whether FPNmediated iron efflux directly regulates iron-dependent proteins and downstream signaling pathways in other cell types, either triggered by changes to hepatic hepcidin or other factors.

Patients with iron overload currently rely on iron chelators and/or phlebotomy to decrease systemic iron levels. However, these therapies often result in suboptimal patient adherence, because iron chelators have off-target effects and chronic phlebotomy can cause fatigue. A selective inhibitor of HIF-2 $\alpha$, PT2385, has recently been developed and is currently in phase II clinical trials for patients with clear-cell renal cell carcinoma (NCT03108066; ClinicalTrials.gov). Here, we sought to address whether oral administration of PT2385 could be used to blunt intestinal iron absorption for the treatment of iron overload. Our data demonstrated that within as little as 2 weeks of PT2385 treatment, systemic iron levels were decreased in mice with established iron overload. This finding provides an exciting impetus for the use of PT2385 in the treatment of human diseases of iron overload, many of which are characterized by dysfunction of the hepcidin/ FPN axis and intestinal iron hyperabsorption.

In conclusion, our work demonstrates that systemic iron deficiency and hepcidin deficiency-mediated iron overload activate the same hepatic hepcidin/intestinal HIF-2 $\alpha$ axis. Moreover, we show that cellular iron efflux through the hepcidin target FPN regulates the activity of iron-dependent enzymes and directly activates HIF-2 $\alpha$. Finally, these data suggest that a therapeutic agent currently in development for humans should be repurposed for the treatment of patients with iron overload.

\section{Methods}

Animals and treatments. For temporal, hepatocyte-specific disruption of hepcidin, mice floxed for Hamp1 $\left(\mathrm{Hamp}^{f / f f}\right)$ on a C57BL/6J background were crossed with $\mathrm{C} 57 \mathrm{BL} / 6 \mathrm{~J}$ mice harboring $\mathrm{Cre}^{E R T 2}$ recombinase under the control of the serum albumin promoter ( $\left.A l b^{\text {CreERT2 }}\right)$ to

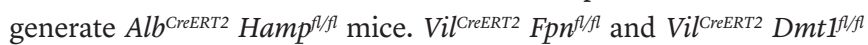
mice are on a 129S4/SvJae background. WT littermates were used as controls for all animal studies (Hamp $p^{f / f l}, F p n^{f / f l}$, and $D m t 1^{f l / f l}$ ), and analysis began on mice that were between 2 and 2.5 months of age for each of the respective experiments. Mice were injected i.p. with tamoxifen (Sigma-Aldrich) at a dose of $100 \mathrm{mg} / \mathrm{kg} \mathrm{BW}$ for 3 consecutive days to ensure Cre-mediated recombination. Phz (Sigma-Aldrich) was administered via i.p. injection at a dose of $60 \mathrm{mg} / \mathrm{kg}$ BW, as described previously (9). PT2385 (MedChemExpress) was prepared and administered daily via oral gavage at a dose of $20 \mathrm{mg} / \mathrm{kg} \mathrm{BW}$, as described previously (45). All mice were fed ad libitum and maintained under a 12-hour light/12-hour dark cycle. All mice were fed either a standard chow diet (Research Diets) or a purified AIN-93G iron-replete (350 ppm) or lowiron ( $<5 \mathrm{ppm})$ diet (Dyets). All mice were housed in the Unit for Laboratory Animal Management (ULAM) at the University of Michigan.

Cell culture. Stable doxycycline-inducible human FPN ${ }^{\text {GFP }}$ HEK293 cells were generated previously (20). To generate stable doxycyclineinducible human FPN ${ }^{\text {GFP }}$ IEC-6 cells, IEC-6 cells were purchased from the American Type Culture Collection (ATCC). pLenti rtTA3 (Addgene) and pLVX-Tight-Puro hFpnGFP plasmids (20) were prepared into lentivirus by the University of Michigan Vector Core, coinfected into IEC- 6 cells, and selected with $10 \mu \mathrm{g} / \mathrm{ml}$ blasticidin and 1 $\mu \mathrm{g} / \mathrm{ml}$ puromycin. Cells were maintained at $37^{\circ} \mathrm{C}$ in $5 \% \mathrm{CO}_{2}$ and $21 \%$ $\mathrm{O}_{2}$. Cells were cultured in DMEM supplemented with $10 \% \mathrm{FBS}$ and $1 \%$ antibiotic/antimycotic. NCOA4-knockout cells were generated 
with the lentiCRISPR v2 construct (Addgene) using 2 unique sgRNAs against NCOA4 (NCOA4 sg1 and NCOA4 sg2, respectively) (Supplemental Table 1). Briefly, oligonucleotides were subcloned into the lentiCRISPRv2 backbone. Empty vector, NCOA4 sg1, and NCOA4 sg2 constructs were prepared into lentivirus by the University of Michigan Vector Core. FPN ${ }^{\text {GFP }}$ cells were infected at a MOI of 10 and selected with $1 \mu \mathrm{g} / \mathrm{ml}$ puromycin. Knockout cells were verified by sequencing. The PHD enzyme activity luciferase reporter was previously described (46). Briefly, FPN ${ }^{\mathrm{GFP}}$ cells were infected at $10 \mathrm{MOI}$ overnight and treated the next day with $250 \mathrm{ng} / \mathrm{ml}$ doxycycline, 100 $\mu$ M FG4592 (Selleckchem), $200 \mu$ M ferric ammonium citrate (FAC) (Sigma-Aldrich), and/or $1 \mathrm{mg} / \mathrm{ml}$ human recombinant hepcidin (Bachem) or $200 \mu \mathrm{M}$ DFO (Sigma-Aldrich). The HIF-2 $\alpha$ IRE luciferase construct was generated previously (24).

Hematological and iron analysis. The Unit for Laboratory Animal Medicine Pathology Core at The University of Michigan performed the complete blood count analysis. Nonheme iron was quantified as described previously (12).

Quantitative reverse transcription PCR. MRNA was measured by quantitative reverse transcription PCR (qPCR) (Life Technologies, Thermo Fisher Scientific). The primers used are listed in Supplemental Table 1. Quantification cycle $(\mathrm{Cq})$ values were normalized to $\beta$-actin and expressed as the fold change.

Whole-genome RNA-Seq and analysis. RNA-Seq libraries were prepared using the TruSeq RNA Library Prep Kit v2 (Illumina) according to the manufacturer's recommended protocol. The libraries were sequenced using single-end, 50-cycle reads on a HiSeq 2500 sequencer (Illumina) at the University of Michigan's DNA Sequencing Core Facility. RNA-Seq analysis was performed as described previously (18). Briefly, quality control of raw FastQ files was performed using FastQC, version 0.11.5. FastQ files were mapped to the mouse genome (mm10) using STAR-2.5.3.a with the options "outFilterMultimapNmax 10" and "sjdbScore 2." Gene expression levels were quantified using the Subread featureCounts (version 1.5.2) package. Differential expression testing was conducted with the Bioconductor package edgeR, version 3.16.5, using glmLRT. To reduce the dispersion of the data set due to lowly expressed genes, genes with a mean aligned read count of less than 5 across all samples were excluded from the analysis. Genes with an FDR of less than 0.01 or 0.1 were considered differentially expressed, to yield high- and low-stringency approaches. The sequencing data are publicly available through ArrayExpress (accession number E-MTAB-7329).

Western blot analysis. Whole-cell, nuclear, and membrane lysates were prepared as described previously $(10,47)$. In brief, lysates were separated by SDS-PAGE, transferred onto nitrocellulose membranes, and probed overnight at $4^{\circ} \mathrm{C}$ with antibodies against FPN (MTP11-A, Alpha Diagnostic International [ADI]), DMT1 (NRAMP21-A, ADI), DCYTB (DCYTB-11A, ADI), and TFR1 (13-6800, Invitrogen, Thermo Fisher Scientific) for mouse tissue lysates; FPN (NBP1-21502, Novus), FTH1 (3998S, Cell Signaling Technology), GAPDH (sc-47724, Santa Cruz Biotechnology), GFP (sc-996, Santa Cruz Biotechnology), lamin
A/C (3A6-4C11, Active Motif), HIF-2 $\alpha$ (BL-95-1A2, Bethyl), and HIF$1 \alpha$ (179483, Abcam) for human lysates; and actin (60008-1, Proteintech) for mouse tissue and rat cell lysates.

ELISA. High-binding polystyrene microtiter plates were coated with the protein lysates overnight. The plates were then washed in $1 \times$ PBS with 0.1\% Tween-20 (PBST), blocked with 5\% BSA, and incubated with primary antibodies against HIF-2 $\alpha$ (AF2997, Novus) and HIF$1 \alpha$ (179483, Abcam). Next, the plates were washed in PBST, incubated with the appropriate HRP-conjugated secondary antibodies, developed, and read at $450 \mathrm{~nm}$ in a plate reader.

Histology, tissue iron staining, and immunohistochemistry. Histologic analysis was performed on H\&E-stained, formalin-fixed, paraffin-embedded sections. Tissue iron detection was performed in formalin-fixed, paraffin-embedded sections stained with Prussian blue. For immunohistochemical analysis, frozen sections were probed with polyclonal rabbit anti-HIF- $2 \alpha$ antibody (100-122, Novus), as previously described (47).

Luciferase assay. Cells were lysed in reporter lysis buffer (Promega), and firefly luciferase activity was measured as described previously (48).

Statistics. Results are expressed as the mean \pm SEM. Significance between 2 groups was tested using a 2-tailed, unpaired $t$ test. Significance among multiple groups was tested using a 1-way or 2-way ANOVA followed by Tukey's post hoc test for multiple comparisons. A $P$ value of less than 0.05 was considered statistically significant. GraphPad Prism 7.0 was used to conduct the statistical analyses.

Study approval. All animal procedures were approved by the IACUC of the University of Michigan.

\section{Author contributions}

AJS and YMS conceived and designed the study. AJS, NKD, SKR, CJ, and MTJ acquired the data. AJS, NKD, SKR, CJ, MTJ, JW, EN, SLL, JAC, and YMS developed the methodologies. AJS, NKD, SKR, CJ, MTJ, JW, EN, SLL, JAC, and YMS analyzed and interpreted the data. AJS and YMS wrote the manuscript. YMS supervised the study.

\section{Acknowledgments}

This work was supported by grants from the NIH (R01CA148828 and R01DK095201, to YMS; F31DK116555, to AJS; K99DK110537, to SKR; R01DK107309, to EN; R01DK107583, to JW; and R01ES028802, to JAC); the American Diabetes Association (1-18IBS-281, to JW); and the University of Michigan GI SPORE Molecular Pathology and Biosample Core (P50CA130810). We would like to thank Matthias Hentze (Heidelberg University, Heidelberg, Germany) for providing the HIF-2 $\alpha$ IRE luciferase construct (24).

Address correspondence to: Yatrik M. Shah, University of Michigan, Department of Molecular and Integrative Physiology, Department of Internal Medicine, Division of Gastroenterology, 7712B MS II, 1301 East Catherine Street, Ann Arbor, Michigan 481095622, USA. Phone: 734.615.0567; Email: shahy@umich.edu.

\footnotetext{
1. McLean E, Cogswell M, Egli I, Wojdyla D, de Benoist B. Worldwide prevalence of anaemia, WHO vitamin and mineral nutrition information system, 1993-2005. Public Health Nutr. 2009;12(4):444-454.

2. Brissot P, Pietrangelo A, Adams PC, de Graaff B,
}

McLaren CE, Loréal O. Haemochromatosis. Nat Rev Dis Primers. 2018;4:18016.

3. Pietrangelo A. Genetics, genetic testing, and management of hemochromatosis: 15 years since hepcidin. Gastroenterology. 2015;149(5):1240-1251.e4.

4. Nemeth E, et al. Hepcidin regulates cel- lular iron efflux by binding to ferroportin and inducing its internalization. Science. 2004;306(5704):2090-2093.

5. Ganz T, Nemeth E. Hepcidin and iron homeostasis. Biochim Biophys Acta. 2012;1823(9):1434-1443.

6. Drakesmith H, Nemeth E, Ganz T. Ironing out 
Ferroportin. Cell Metab. 2015;22(5):777-787.

7. Mastrogiannaki M, Matak P, Keith B, Simon MC, Vaulont S, Peyssonnaux C. HIF-2alpha, but not HIF-1alpha, promotes iron absorption in mice. JClin Invest. 2009;119(5):1159-1166.

8. Shah YM, Matsubara T, Ito S, Yim SH, Gonzalez FJ. Intestinal hypoxia-inducible transcription factors are essential for iron absorption following iron deficiency. Cell Metab. 2009;9(2):152-164.

9. Anderson ER, Xue X, Shah YM. Intestinal hypoxia-inducible factor-2alpha (HIF-2alpha) is critical for efficient erythropoiesis. J Biol Chem. 2011;286(22):19533-19540.

10. Taylor M, et al. Hypoxia-inducible factor- $2 \alpha$ mediates the adaptive increase of intestinal ferroportin during iron deficiency in mice. Gastroenterology. 2011;140(7):2044-2055.

11. Ramakrishnan SK, Anderson ER, Martin A, Centofanti B, Shah YM. Maternal intestinal HIF-2 $\alpha$ is necessary for sensing iron demands of lactation in mice. Proc Natl Acad Sci U S A. 2015;112(28):E3738-E3747.

12. Das N, Xie L, Ramakrishnan SK, Campbell A, Rivella S, Shah YM. Intestine-specific disruption of hypoxia-inducible factor (HIF)-2 $\alpha$ improves anemia in sickle cell disease. J Biol Chem. 2015;290(39):23523-23527.

13. Anderson ER, et al. Intestinal HIF $2 \alpha$ promotes tissue-iron accumulation in disorders of iron overload with anemia. Proc Natl Acad Sci U S A. 2013;110(50):E4922-E4930.

14. Mastrogiannaki M, Matak P, Delga S, Deschemin JC, Vaulont S, Peyssonnaux C. Deletion of HIF$2 \alpha$ in the enterocytes decreases the severity of tissue iron loading in hepcidin knockout mice. Blood. 2012;119(2):587-590.

15. Berdoukas V, Coates TD, Cabantchik ZI. Iron and oxidative stress in cardiomyopathy in thalassemia. Free Radic Biol Med. 2015;88(Pt A):3-9.

16. Lakhal-Littleton $S$, et al. Cardiac ferroportin regulates cellular iron homeostasis and is important for cardiac function. Proc Natl Acad Sci U S A. 2015;112(10):3164-3169.

17. Derchi G, et al. Clinical management of cardiovascular complications in patients with thalassaemia major: a large observational multicenter study. Eur J Echocardiogr. 2011;12(3):242-246.

18. Triner D, Xue X, Schwartz AJ, Jung I, Colacino JA, Shah YM. Epithelial hypoxia-inducible factor $2 \alpha$ facilitates the progression of colon tumors through recruiting neutrophils. Mol Cell Biol. 2017;37(5):e00481-16.

19. Xue X, Ramakrishnan SK, Shah YM. Activation of HIF-1 $\alpha$ does not increase intestinal tumorigenesis. Am J Physiol Gastrointest Liver Physiol. 2014;307(2):G187-G195.
20. Qiao B, et al. Hepcidin-induced endocytosis of ferroportin is dependent on ferroportin ubiquitination. Cell Metab. 2012;15(6):918-924.

21. Yoon YS, et al. Formation of elongated giant mitochondria in DFO-induced cellular senescence: involvement of enhanced fusion process through modulation of Fis1. JCell Physiol. 2006;209(2):468-480.

22. Yoon YS, Cho H, Lee JH, Yoon G. Mitochondrial dysfunction via disruption of complex II activity during iron chelation-induced senescence-like growth arrest of Chang cells. Ann N Y Acad Sci. 2004;1011:123-132.

23. Chandel NS, et al. Reactive oxygen species generated at mitochondrial complex III stabilize hypoxia-inducible factor-1alpha during hypoxia: a mechanism of $\mathrm{O} 2$ sensing. J Biol Chem. 2000;275(33):25130-25138.

24. Sanchez M, Galy B, Muckenthaler MU, Hentze MW. Iron-regulatory proteins limit hypoxiainducible factor-2alpha expression in iron deficiency. Nat Struct Mol Biol. 2007;14(5):420-426.

25. Mancias JD, et al. Ferritinophagy via NCOA4 is required for erythropoiesis and is regulated by iron dependent HERC2-mediated proteolysis. Elife. 2015;4:e10308.

26. Mancias JD, Wang X, Gygi SP, Harper JW, Kimmelman AC. Quantitative proteomics identifies NCOA4 as the cargo receptor mediating ferritinophagy. Nature. 2014;509(7498):105-109.

27. Ross SL, et al. Molecular mechanism of hepcidin-mediated ferroportin internalization requires ferroportin lysines, not tyrosines or JAK-STAT. Cell Metab. 2012;15(6):905-917.

28. Deshpande CN, et al. Calcium is an essential cofactor for metal efflux by the ferroportin transporter family. Nat Commun. 2018;9(1):3075.

29. Wallace EM, et al. A small-molecule antagonist of HIF2 $\alpha$ is efficacious in preclinical models of renal cell carcinoma. Cancer Res. 2016;76(18):5491-5500.

30. Kelly CJ, et al. Crosstalk between microbiotaderived short-chain fatty acids and intestinal epithelial HIF augments tissue barrier function. Cell Host Microbe. 2015;17(5):662-671.

31. Lakhal-Littleton S, et al. An essential cell-autonomous role for hepcidin in cardiac iron homeostasis. Elife. 2016;5:e19804.

32. Guo S, et al. Reducing TMPRSS6 ameliorates hemochromatosis and $\beta$-thalassemia in mice. JClin Invest. 2013;123(4):1531-1541.

33. Kautz L, Jung G, Valore EV, Rivella S, Nemeth $\mathrm{E}, \mathrm{Ganz}$ T. Identification of erythroferrone as an erythroid regulator of iron metabolism. Nat Genet. 2014;46(7):678-684.

34. Tanno T, et al. High levels of GDF15 in thalassemia suppress expression of the iron regulatory protein hepcidin. Nat Med.2007;13(9):1096-1101.

35. Tanno $\mathrm{T}$, et al. Identification of TWSG1 as a second novel erythroid regulator of hepcidin expression in murine and human cells. Blood. 2009;114(1):181-186.

36. Taniguchi CM, et al. Cross-talk between hypoxia and insulin signaling through Phd3 regulates hepatic glucose and lipid metabolism and ameliorates diabetes. Nat Med. 2013;19(10):1325-1330.

37. Taniguchi CM, et al. PHD inhibition mitigates and protects against radiation-induced gastrointestinal toxicity via HIF2. Sci Transl Med. 2014;6(236):236ra64.

38. Zhang DL, Hughes RM, Ollivierre-Wilson H, Ghosh MC, Rouault TA. A ferroportin transcript that lacks an iron-responsive element enables duodenal and erythroid precursor cells to evade translational repression. Cell Metab. 2009;9(5):461-473.

39. Anderson SA, et al. The IRP1-HIF-2 $\alpha$ axis coordinates iron and oxygen sensing with erythropoiesis and iron absorption. Cell Metab. 2013;17(2):282-290.

40. Ghosh MC, et al. Deletion of iron regulatory protein 1 causes polycythemia and pulmonary hypertension in mice through translational derepression of HIF2 $\alpha$. Cell Metab. 2013;17(2):271-281.

41. Wilkinson N, Pantopoulos K. IRP1 regulates erythropoiesis and systemic iron homeostasis by controlling HIF $2 \alpha$ mRNA translation. Blood. 2013;122(9):1658-1668.

42. Ghosh MC, Zhang DL, Ollivierre H, Eckhaus MA, Rouault TA. Translational repression of HIF2 $\alpha$ expression in mice with Chuvash polycythemia reverses polycythemia. JClin Invest. 2018;128(4):1317-1325.

43. Britton L, et al. Ferroportin expression in adipocytes does not contribute to ironhomeostasis or metabolic responses to a high calorie diet. Cell Mol Gastroenterol Hepatol. 2018;5(3):319-331.

44. Wu LJ, et al. Expression of the iron transporter ferroportin in synaptic vesicles and the bloodbrain barrier. Brain Res. 2004;1001(1-2):108-117.

45. Xie C, et al. Activation of intestinal hypoxia-inducible factor $2 \alpha$ during obesity contributes to hepatic steatosis. Nat Med. 2017;23(11):1298-1308.

46. Kim DI, et al. An OLTAM system for analysis of brown/beige fat thermogenic activity. Int JObes (Lond). 2018;42(4):939-945.

47. Ramakrishnan SK, et al. HIF2 $\alpha$ Is an essential molecular brake for postprandial hepatic glucagon response independent of insulin signaling. Cell Metab. 2016;23(3):505-516.

48. Xue X, et al. Iron uptake via DMT1 integrates cell cycle with JAK-STAT3 signaling to promote colorectal tumorigenesis. Cell Metab. 2016;24(3):447-461. 